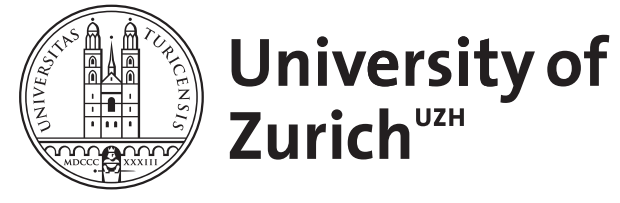
Archive

University of Zurich

University Library

Strickhofstrasse 39

CH-8057 Zurich

www.zora.uzh.ch

Year: 2012

\title{
Guillaume de Leus, commentateur du Liber de causis
}

\author{
Carron Faivre, Delphine
}

DOI: https://doi.org/10.1484/J.BPM.1.103408

Posted at the Zurich Open Repository and Archive, University of Zurich

ZORA URL: https://doi.org/10.5167/uzh-108440

Journal Article

Originally published at:

Carron Faivre, Delphine (2012). Guillaume de Leus, commentateur du Liber de causis. Bulletin de Philosophie Médiévale, 54:297-331.

DOI: https://doi.org/10.1484/J.BPM.1.103408 


\section{GUILLAUME DE LEUS, COMMENTATEUR DU LIBER DE CAUSIS}

\section{L'auteur du commentaire : Guillaume de Leus}

C'est Auguste Pelzer, dans une notice rédigée en 1935, qui mit le premier en lumière la carrière et les œuvres du frère prêcheur Guillaume de Leus, en complétant les données rassemblées par Célestin Douais à la fin du XIX siècle ${ }^{1}$. Depuis lors, quelques travaux se sont intéressés à la vie et à l'œuvre du dominicain : la notice de Maurice de Wulf dans son Histoire de la philosophie médiévale ${ }^{2}$, celle de Charles Lohr dans son inventaire des commentateurs d'Aristote ${ }^{3}$, celle de Thomas Kaeppeli pour ses Scriptores Ordinis

Nous aimerions à cette occasion témoigner notre reconnaissance à Ruedi Imbach, qui dans le cadre d'une bourse postdoctorale de six mois en 2011 (liée au projet THOM ANRDFG), nous a encouragée à travailler sur ce commentaire du Liber de Causis et nous a accompagnée dans cette découverte. Que Dragos Calma, qui nous a invitée à collaborer avec son équipe de l'Université de Cluj et avec qui nous avons eu de fructueuses discussions concernant cet article, William Duba, avec qui nous avons réfléchi sur de nombreux points philologiques et historiques, et Martin Morard, qui nous a fait part de plusieurs suggestions terminologiques et bibliographiques, soient ici vivement remerciés.

${ }^{1}$ Cf. A. Pelzer, «Guillaume de Leus (de Levibus) frère prêcheur de Toulouse », dans Aus der Geisteswelt des Mittelalters. Studien und Texte Martin Grabmann zur Vollendung des 60. Lebensjahres von Freuden und Schülern gewidmet, hrsg. v. A. LANG, J. LECHNER und M. SCHMAUS, Münster i.W. 1935, 1065-79, réimprimé dans Études d'histoire littéraire sur la scolastique médiévale, éd. A. PATTIN et E. VAN DE VyVER, Louvain-Paris 1964, 392410 (nous citons les pages de la réimpression). Voir aussi les écrits de C. DoUAIs, Essai sur l'organisation des études dans l'ordre des frères prêcheurs au treizième et au quatorzième siècle (1216-1342), Paris-Toulouse 1884 ; Les frères prêcheurs en Gascogne au XIII siècle et au XIV siècle, Paris-Auch 1885 ; ed., Acta capitulorum provincialium ordinis fratrum praedicatorum. Première province de Provence, province romaine, province d'Espagne (1239-1302), Toulouse 1894. Dans son article, Pelzer fait aussi référence au Répertoire des maîtres en théologie de Paris au XIII siècle de P. GLORIEUX (t. 1, Paris 1933, 213 n 69) où Guillaume de Leus est cependant confondu avec un Guillaume de Lhuys, socius de Gui de la Tour, évêque de Clermont; à l'Historia bibliothecae romanorum pontificum tum Bonifatianae tum Avenionensis de F. EHRLE (t. 1, Città del Vaticano 1890, 321 et 397) ainsi qu'à deux articles de M. GRABMANN, «De summae Thomae Aquinatis theologicae studio in ordine fratrum praedicatorum iam saeculis XIII et XIV vigente », dans Miscellanea dominicana in memoriam VII anni saecularis ab obitu s. patris Dominici (1221-1921), Roma 1923, 151-61, ici 160 ; «Die Proklosübersetzungen des Wilhelm von Moerbeke und ihre Verwertung in der lateinischen Literatur des Mittelalters », dans Byzantinische Zeitschrift 30 (1929-1930), 78-88, ici 85).

${ }^{2}$ M. DE WULF, Histoire de la philosophie médiévale 3 : Après le XIII siècle, Paris 1947, 61.

${ }^{3}$ C. LoHR, « Medieval Latin Aristotle Commentaries: Authors G-I », dans Traditio 24 Bulletin de philosophie médiévale 54 (2012), 297-331. DOI: 10.1484/J.BPM.1.103408 (C) 2013, Brepols Publishers, n.v. All rights reserved. 
Praedicatorum Medii Aevi ${ }^{4}$, celle d'Olga Weijers dans son répertoire sur les maîtres de la Facultés des Arts de Paris (dans la catégorie S : sources contemporaines relatives aux disciplines enseignées) ${ }^{5}$, ainsi que celle du registre Personennamen des Mittelalters ${ }^{6}$.

Cet auteur figure dans les documents de son époque sous des appellations variées. Les trois codex qui renferment - à notre connaissance - ses écrits, les manuscrits de la Bibliotheca Apostolica Vaticana, Codd. Borgh. 165 et 352 ainsi que celui de la cathédrale de Tortosa, Cód. 229, le nomment respectivement dans les épîtres dédicatoires "Guillelmus de Lieus" (f. 1ra) et "Guillelmus de Liues» (f. 1ra), ainsi que dans l'explicit "Guillelmus de Liens » (f. 82r). Il est aussi question de «Guillelmus de Lyens» dans une lettre de Clément $\mathrm{V}^{7}$. Dans la liste intitulée Magistri in theologia Parisius, on lit : « frater Guillelmus de Leus, tholosanus, per dominum papam Clementem $\mathrm{V}$ in Tholosa... $»^{8}$. Quant au chapitre provincial de Bordeaux de 1311, il parle de $"$ frater Guillermus de Leus »". Les autres relations des chapitres provinciaux décrivent les charges successives d'enseignement d'un « Guillelmus » ou «Willelmus » ou encore «Guillermus de Levibus ${ }^{10}$. Enfin, parmi les inventaires de la bibliothèque d'Avignon, celui de 1369 parle d'un « G. de Liens », celui de 1411 d'un " Guillelmus de Lieus », et celui de 1594 d'un « Guillermus de Lives » ${ }^{11}$. On ne peut ainsi que relever l'indécision qui règne

(1968), 149-245, ici 201-2.

${ }^{4}$ T. KAEPPELI, « Guillelmus de Levibus (Leus) Tolosanus », dans Scriptores Ordinis Praedicatorum Medii Aevi 2, Roma 1975, 108-9, présente deux nouveaux éléments importants : la dernière information biographique connue, concernant sa nomination épiscopale et une nouvelle œuvre attribuée, le Tractatus de predestinacione.

${ }^{5}$ O. WeIJERs, Le travail intellectuel à la Faculté des Arts de Paris : textes et maîtres (ca. 1200-1500) 3 (Studia Artistarum 6), Turnhout 1998, 118-19. O. Weijers explique : «Son commentaire sur le De causis, bien que postérieur à sa période parisienne, fut probablement connu à Paris ».

${ }^{6}$ Personennamen des Mittelalters, hrsg. v. C. FABIAN, München $2000^{2}, 252$ col. 2.

${ }^{7}$ Città del Vaticano, Bibliotheca Apostolica Vaticana, Cod. Regin. lat., 56, f. 4v. Voir aussi H. Denifle et A. Chatelain, Chartularium Universitatis Parisiensis II, Paris 1891, $137 \mathrm{n}^{\circ} 674$.

${ }^{8}$ Liste commencée par Étienne de Salanhac, continuée par Bernard Gui et d'autres, éditée par H. DeNIFLE, «Quellen zur Gelehrtengeschichte des Predigerordens im 13. und 14. Jahrhundert », dans Archiv für Literatur- und Kirchengeschichte des Mittelalters 2 (1886), 165248, ici $214 \mathrm{n}^{\circ} 62$.

${ }^{9}$ Voir infra, n. 38.

${ }^{10}$ Voir infra, nn. 18-31.

${ }^{11}$ Cf. respectivement F. EHRLE, « Recensio librorum anno 1369 in palatio Avenionensi asservatorum », dans Historia bibliothecae Romanorum pontificium tum Bonfitianae tum 
à propos de l'orthographe du nom de l'auteur ${ }^{12}$. Il se pourrait cependant que le frère prêcheur provienne d'une des nombreuses branches de la maison de Lévis, installée à partir de 1229 dans le fief de Mirepoix (depuis que Gui I ${ }^{\text {er }}$ de Lévis s'illustra durant la croisade contre les Albigeois), à une quarantaine de kilomètres de Toulouse ${ }^{13}$.

\section{Sa vie}

Guillaume de Leus, toulousain par sa naissance et sa filiation religieuse ${ }^{14}$, a connu un beau parcours scientifique et ecclésiastique, avant tout dans cette province dominicaine de Provence, où a été fondé (en 1216 à Toulouse) le premier couvent, berceau de l'Ordre des frères prêcheurs ${ }^{15}$. Le pape Clément $\mathrm{V}$, dans une lettre du 6 janvier 1309, en rappelle les étapes essentielles :

frater Guillelmus de Lyens... diu institerit studio sciencie memorate [theologie] et perstiterit per Dei graciam adeo laudabiliter in eadem quod in studiis generalibus et pluribus locis insignibus libros dicte sciencie solenniter in huiusmodi studiis consuetos legerit ac alios instruxerit ad

Avenionensis 1, 277-450, 321 ; A. MAIER, « Der Katalog der päpstlichen Bibliothek in Avignon vom Jahr 1411 », et «Der letze Katalog der päpstlichen Bibliothek von Avignon (1594) », dans Ausgehendes Mittelalter. Gesammelte Aufsätze zur Geistesgeschichte des 14. Jahrhundert III, hrsg. v. A. PARAVICINI BAGLIANI (Storia e letteratura 138), Roma 1977, $77-$ 157, ici 154, et 187-248, ici 224.

${ }^{12}$ Relevons cependant qu'il s'agit avant tout d'une confusion entre les lettres $u$ et $n$, très fréquente dans la lecture des manuscrits et d'une inversion des lettres eu et $u e$.

${ }^{13}$ Nous remercions à ce propos Zénon Kaluza, qui a proposé ce lien avec la famille des Lévis. G. MARTIN, Histoire et généalogie de la Maison de Lévis, Lyon 2007, ne mentionne pas un Guillaume ayant vécu au XIII ${ }^{\mathrm{e}}$ siècle parmi ses généalogies, mais celles-ci sont forcément lacunaires. Il parle en revanche, 239, « d'autres Lévis » qu'il n'a pu classer, et parmi eux d'un certain Durand de Lévis, consul de Toulouse en 1226. PELzer, "Guillaume de Leus (de Levibus) frère prêcheur de Toulouse », 395, fait en outre le lien entre les orthographes Levibus et Levis, en citant l'Histoire générale du Languedoc VIII, de D. DEVIC et D. Vaissete, de l'édition Privat, qui mentionne un Guillaume et un Pierre de Leus, l'un consul à Toulouse, l'autre témoin dans une sommation, et qui renvoie dans l'Index onomastique à « Levibus, Levis ».

${ }^{14}$ Dans les deux épîtres dédicatoires que nous possédons (Città del Vaticano, BAV, Cod. Borgh. 352, ff. 1ra et 165, f. 1ra), Guillaume se décrit lui-même comme « tholosanus nacione ».

${ }^{15}$ Cette première province dominicaine de Provence sera scindée en deux en 1303, en (seconde) province de Provence et Province de Toulouse. Voir à ce propos DouAIS, Essai, XXI et 4-5, 92, 236. On pourrait même ajouter que la carrière de Guillaume de Leus, outre ses années d'étude à Paris, s'est complètement déroulée dans ce que le chapitre provincial de 1271 nomme in media parte provincie, à savoir les couvents de Béziers, Narbonne, Perpignan, Carcassone, Castres, Nîmes, Montpellier, Toulouse, Montauban et Cahors. 
doctrinam ${ }^{16}$.

Sa carrière nous est connue en détail grâce aux chapitres annuels provinciaux, édités en partie et compulsés par C. Douais, puis repris par A. Pel$z^{2}{ }^{17}$. Même si l'on a aucune information concernant des études préliminaires en logique et en philosophie - que tout jeune dominicain promis à un bel avenir scientifique accomplissait -, on peut supposer que Guillaume de Leus commence sa formation dans les studia arcium et naturarum des couvents dominicains, ces informations étant d'ailleurs rarement mentionnées dans les Actes des chapitres provinciaux avant 1280.

Ce dont on est par contre certain, c'est que Guillaume de Leus est assigné pour les années 1281-1282 à l'étude de la théologie au couvent de Béziers $^{18}$. Il est ensuite directement désigné comme sous-lecteur de théologie, sous l'autorité du lecteur frère Jordanus, au studium solemne de Toulouse pour les années 1283-1284 ${ }^{19}$, puis lecteur en 1285 à Castres, et ce déjà avec la faculté de disputer, qui n'est accordée qu'aux lecteurs les plus capables et les plus habiles ${ }^{20}$.

En juillet 1286, le chapitre provincial de Bergerac le choisit parmi les trois étudiants qu'il envoie au studium generale de Paris ${ }^{21}$. À son retour, Guillaume est nommé lecteur de théologie à Narbonne pour les années

\footnotetext{
${ }^{16}$ BAV, Cod. Regin. lat. 56, f. 4v. Voir aussi Denifle et Chatelain, Chartularium Universitatis Parisiensis II, 137.

${ }^{17}$ Célestin Douais a édité les Actes des chapitres jusqu'en 1302 dans les Acta capitulorum provincialium ordinis fratrum praedicatorum. Première province de Provence, province romaine, province d'Espagne (1239-1302). Voir aussi PELZER, « Guillaume de Leus (de Levibus) frère prêcheur de Toulouse », 395-96.

${ }^{18}$ Acta, ed. DouAis, 250 (1281, Marseille) : «Assignamus studentes [mg. studentes theologie].... Bitterris, fratres G. de Levibus, Castellum Novum, Ber. de Birano, Dominicum Bitterrensem ».

${ }^{19}$ Acta, ed. DouAIs, 266 (1283, Montpellier) : «Ad secundam leccionem assignamus lectores [mg. sublectores] : ...Tholose, fratrem Guillermum de Levibus ». Selon DoUAIS, Essai, 126, les studia solemnia sont des écoles de haut enseignement théologique, réservées aux meilleurs élèves de la province, où l'on fait la plus large application possible de la philosophie à la théologie, selon la méthode des scolastiques.

${ }^{20}$ Acta, ed. DouAis, 285 (1285, Condom) : «Assignamus lectores theologie.... Castris, fratrem $W^{\text {um }}$ de Levibus ; et disputet ». Pour des informations sur cette faculté de disputer, voir DouAIs, Essai, 77-78, qui ajoute que les Actes des chapitres provinciaux prescrivent régulièrement aux lecteurs en théologie de disputer au moins une fois chaque quinzaine, et ce de manière publique.

${ }^{21}$ Acta, ed. DouAis, 298 (1286, Bergerac) : « Studentes mittendos Parisius pro anno sequenti assignamus fratres P. Tardivi Diensem, $\mathrm{W}^{\mathrm{m}}$ de Levibus Tholosanum, G. de Malartic Baionensem ».
} 
1288-1289 ${ }^{22}$, puis à Carcassonne en 1290 , en la même qualité mais avec la faculté de disputer ${ }^{23}$. Élevé au titre de prédicateur général en $1291^{24}$ et ainsi autorisé à exercer le ministère au-delà des limites d'un couvent ${ }^{25}$, il est à nouveau lecteur à Narbonne en $1292^{26}$, puis sous-lecteur au studium generale de Montpellier en 1293-1294, sous l'autorité du lecteur Guillaume de Quinssaco, demeuré à sa fonction durant presque trente ans ${ }^{27}$. Il est nommé lector Biblie au studium solemne de Toulouse en 1295, sous l'autorité d'un lecteur, maître en théologie, Raymond Guilha ${ }^{28}$. Il est une troisième fois lecteur à Narbonne pour les années $1296-1300^{29}$, puis lecteur à Carcassonne en $1300^{30}$ et à nouveau prédicateur général en $1302^{31}$. Enfin, en 1303, Guillaume est désigné comme lecteur du couvent de Toulouse ${ }^{32}$ - que le cha-

${ }^{22}$ Acta, ed. DouAis, 312 (1288, Avignon) : « Assignamus lectores in theologia.... Narbone, lectorem fratrem G. de Levibus ».

${ }^{23}$ Acta, ed. DouAIS, 332-33 (1290, Pamiers) : «Assignamus lectores fratres [mg. lectores theologie].... Carcassone, $\mathrm{W}^{\mathrm{m}}$ de Levibus ; et disputet ».

${ }^{24}$ Acta, ed. DouAIs, 356 (1291, Béziers) : «Faciamus predicatores generales fratres... G. de Levibus ».

${ }^{25}$ DouAIs, Essai, 13-14 : «Seuls les prédicateurs généraux, predicatores generales, toujours désignés par le chapitre provincial et en petit nombre, étaient autorisés à déployer leur zèle au-delà de ces limites [i.e. celles d'une Prédication, ministère extérieur d'un couvent qui embrasse une certaine étendue géographique, dont l'autorité du chapitre provincial fixe les limites] : seuls ils pouvaient se faire entendre dans les grande villes, dans les "chaires solennelles" ».

${ }^{26}$ Acta, ed. DouAis, 360 (1292, Brive) : « Assignamus lectores, fratres [mg. lectores theologie].... Narbone, G. de Levibus ».

${ }^{27}$ Acta, ed. DouAIs, 374 (1293, Carcassonne) : «Ad secundam leccionem fratres. In Montepessulano, $\mathrm{W}^{\mathrm{m}}$ de Levibus ». Les studia generalia ressemblent, quant au contenu de l'enseignement, aux studia solemnia, bien que d'un niveau encore supérieur, mais sont surtout différents quant à leur rôle : ils sont pour l'Ordre tout entier, et réunissent les meilleurs éléments des différentes provinces. Voir à ce propos DouAIS, Essai, 130-31.

${ }^{28}$ Acta, ed. DouAIS, 396 (1295, Castres) : «Ad legendam Bibliam : Conventui Tholosano, fratrem G. de Levibus ». Concernant Raymond Guilha, cf. Ibid., 395 : «Ad promocionem studii Tholosani, reverendum patrem fratrem Ramundum Guilha, professorem theologie facultatis, assignamus pro doctore conventui Tholosano ", et DouAIs, Les frêres prêcheurs, 474.

${ }^{29}$ Acta, ed. DouAis, 403 (1296, Narbonne) : «Assignamus lectores fratres.... Narbone, Guillermum de Levibus ». Sous son autorité, trois sous-lecteurs vont se succéder: fr. P. Amati (1296), fr. Andricus Maurandi (1297) et fr. G. Fabri de Alzona (1299). Voir à ce propos DouAIs, Essai, 226.

${ }^{30}$ Acta, ed. DouAIS, 450 (1300, Marseille) : «Assignamus lectores theologie fratres.... Carcassone, $\mathrm{W}^{\mathrm{m}}$ de Levibus "».

${ }^{31}$ Acta, ed. DouAIs, 479 (1302, Carcassone) : « Faciamus predicatores generales fratres... $\mathrm{W}^{\mathrm{m}}$ de Levibus ».

${ }^{32}$ À partir de cette date, les Actes ne sont plus édités, il faut donc se reporter au ms. Tou- 
pitre général de 1304 fixe comme nouveau studium generale ${ }^{33}$.

En janvier 1309, le pape Clément V séjournant à Toulouse - lui-même, d'ailleurs, ancien de la faculté de droit de l'Université de cette ville - accorde au frère Guillaume de Leus, à travers une lettre adressée au cardinal Pierre de la Chapelle-Taillefer, la faveur quasi-inédite ${ }^{34}$ d'être admis à la licencia ubicumque docendi en théologie, sur place, sans passer par l'Université de Paris :

nos hiis debita consideracione pensatis dignum fore putavimus et consentaneum racioni, ut idem frater Guillelmus illa favorabiliter gracia honoretur ad quam ydoneus comprobatur ob suorum exigenciam meritorum. De tua [frater Petrus episcopus Penestrinus] itaque circumspeccione plenam in Domino fiduciam obtinentes fraternitati tue licenciandi eundem fratrem Guillelmum auctoritate nostra in prefata sciencia theologie et concedendi ei licenciam legendi in illa cum plenitudine magisterii sibique demum sic licenciato incipiendi legere in prefata sciencia ubicunque voluerit ordinarie, ut est moris, plenam et liberam auctoritate apostolica concedimus potestatem ${ }^{35}$.

Suite à cette promotion, Guillaume semble ne pas conserver sa fonction au couvent de Toulouse et le chapitre de Périgueux de 1309 désigne un nouveau lecteur en théologie en la personne d'Arnaud de Barriera ${ }^{36}$. Quant à

louse, BM, 490 ou aux extraits publiés par C. Douais. Pour ce passage, le manuscrit, f. 387r nous est resté illisible ; voir donc DouAIS, Essai, 236: «1303. fr. W. de Levibus ». Guillaume aura sous son autorité un sous-lecteur, des lectores Biblie et lectores Sentenciarum. Comme sous-lecteur : 1303-1309, fr. Jo. de Faubeto ; comme lecteurs de la Bible : 13031307, fr. P. Bernardi et 1307-1309, fr. Aymericus de Miromonte ; comme lecteurs des Sentences : 1303-1307, fr. $W^{\mathrm{m}}$ Petri de Godino, 1307, Jordanus de Castro Novo et 1308, fr. Ber. de Malartico. Voir à ce propos DouAIS, Essai, 236, 271-72.

${ }^{33}$ Cf. DouAIs, Essai, 127, 236, qui cite le ms. Toulouse, BM, 490, f. 390r : « Assignamus locum studii generalis conventum tholosanum ».

${ }^{34}$ Nous avons lu dans la liste des Maîtres dominicains parisiens, réalisée par Étienne de Salanhac et Bernard Gui, que Rémi de Florence avait déjà obtenu cette faveur en 1302 grâce à Boniface VIII, texte que l'on peut lire entre autres chez DoUAIS, Essai, 166: «Frater Remigius Florentinus, licenciatus per Bonifacium papam VIII, anno Domini $\mathrm{M}^{\circ} \mathrm{CCC}^{0} \mathrm{II}^{0} »$. Un autre dominicain, frère Raymond de Corsavino, recevra d'ailleurs de Clément V la même faveur pour les studia de Montpellier ou Toulouse, quelques années plus tard, en 1313. Voir à ce propos S. PIRON, «Les studia franciscains de Provence et d'Aquitaine (12751335) ", dans Philosophy and Theology in the Studia of the Religious Orders and at Papal and Royal Courts, ed. K. EMERY, Jr., W.J. CourtenAy and S.M. Metzger (Rencontres de philosophie médiévale 15), Turnhout 2012, 303-58, ici 326, qui fait référence au Chartularium Universitatis Parisiensis II, 168.

${ }^{35}$ Comme nous l'avons déjà signalé en notes 8 et 17, la lettre peut se lire dans le ms. BAV, Cod. Regin. lat. 56, f. 4v, et est publiée dans le Chartularium II, 137.

${ }^{36}$ Toulouse, BM, Ms. 490, f. 407r (in calce pagine) : « frater Arnaldus de Barreria, lector Tholosanus ». Ni Douais ni Pelzer n'avait relevé cette information, qui suggère que Guil- 
Guillaume de Leus, on ne sait s'il demeure à Toulouse durant les deux années suivantes. L'élément certain est que le chapitre provincial tenu à Bordeaux le 15 août 1311 ordonne solennellement à Guillaume de Leus de donner, lorsqu'il sera de retour à Toulouse(!), sa leçon inaugurale et de disputer de manière ordinaire et quodlibétique, en tant que nouveau maître en théologie, pour l'honneur et la promotion de ce studium ${ }^{37}$ :

Ad honorem et promocionem studii tholosani, taliter ordinamus, quod cum frater Guillermus de Leus, magister in theologia, apud Tholosam pervenerit, ibidem incipiat et disputet de quodlibet et aliis, prout sibi visum fuerit, ut magister; et nichilominus assignamus lectorem fratrem Dominicum Grimam ${ }^{38}$.

Un nouveau lecteur est aussi nommé pour le couvent, Dominique Grima (ou Grenier), promis à une très belle carrière ${ }^{39}$. Guillaume n'exerce finalement comme magister que deux ans, puisqu'il est nommé évêque de Termoli en 1313, et célèbre sa première messe épiscopale en présence du roi de Naples Robert $1^{\text {er }} \mathrm{d}^{\prime}$ Anjou ${ }^{40}$. Ce sont les dernières informations que nous possédons sur sa vie.

\section{Son œuvre}

Selon le témoignage des deux manuscrits de la Bibliothèque Vaticane respectivement Codd. Borgh. 352 et 165 -, Guillaume de Leus dédie au cardinal Pierre de la Chapelle-Taillefer, ancien évêque de Toulouse (1298-

laume n'est plus lecteur du couvent de Toulouse à partir de 1309.

${ }^{37}$ Ce studium pourrait aussi se comprendre comme l'intégralité de la communauté universitaire, à savoir la communauté toulousaine des magistri. Voir à ce propos la discussion concernant l'Université de Toulouse, infra, 307-8.

${ }^{38}$ Toulouse, BM, Ms. 490, f. 410v. Le texte est évidemment aussi cité par DouAIS, par exemple dans Essai, 236. À nouveau, même lorsque Guillaume revient en 1311 à Toulouse pour exercer en tant que magister theologie, il ne recouvre pas la charge de lector theologie.

${ }^{39}$ Il sera maître du Sacré Palais et maître en théologie, lui aussi par mandat pontifical (Jean XXII), enfin évêque de Pamiers. Voir à ce propos DouAIS, Les frères prêcheurs, 401, et surtout M. MorARD, «Dominique Grima, o. p., un exégète thomiste à Toulouse au début du XIV siècle », dans Église et culture en France méridionale (XII ${ }^{e}-X I V^{e}$ siècles) : Cahiers de Fanjeaux 35 (2000), 325-74, en part. 326-27 et 343. Concernant la fonction de maître du Sacré Palais, voir M. MORARD, « Le studium de la Curie pontificale et ses maîtres au temps de Jean XXII », dans Jean XXII et le Midi : Cahiers de Fanjeaux 45 (2010), 461-545, en part. 516-19.

${ }^{40}$ N. BARONE, «La Ratio Thesaurariorum della Cancelleria angioina, 1312-1325 », dans Archivio storico per le provincie napoletane 11 (1886), 175-97, ici 176: «Anno 1313, Marzo, 18: Roberto fa pagare a Gerìo Ardinghelli, mercatante della società dei Bardi di Firenze, 2 once e 15 tarì per una coppa di argento dorato, senza coperchio, del peso di 16 once e mezzo, la quale egli stesso dà in dono a Frate Guglielmo de Leus, vescovo di Termoli, che in questo dì, alla presenza di lui, celebra novam missam episcopalem ». 
1305), nommé cardinal de Palestrina par Clément V, une Explication du Livre des Causes et une Explication des six Décrétales accompagnée - afin de prolonger les réflexions des trois dernières décrétales ${ }^{41}$ - d'une Explication de la Messe et d'une Histoire de la Passion du Christ.

Outre ces quatre écrits, nous connaissons encore un autre ouvrage contenu dans le codex Tortosa, Biblioteca del Cabildo, Cód. 229 (ff. 6282), intitulé Traité de la prédestination des élus et de la réprobation des méchants, composé alors que Guillaume est lecteur à Toulouse ${ }^{42}$.

Enfin, nous savons que le dominicain a rédigé un commentaire des trois premiers livres des Sentences - auquel il fait plusieurs fois référence dans ses deux autres commentaires-, mais que pour l'instant nous n'avons pas retrouvé $e^{43}$.

S'il n'est pas difficile d'imaginer le frère prêcheur Guillaume de Leus composant ces trois petits traités théologiques et lisant les Sentences, les contextes dans lesquels ont été réalisés ses commentaires aux Décrétales et au Livre des Causes exigent que l'on s'y arrête quelques instants.

En ce qui concerne l'Exposicio sex Decretalium, l'affaire semble assez claire : en tant que lecteur du couvent de Toulouse, comme il le dit luimême dans la dédicace au cardinal de la Chapelle, Guillaume de Leus a réalisé un exercice qui est une "véritable spécialité toulousaine " ${ }^{44}$, à savoir des leçons données les jours de fête sur les Décrétales à caractère théo-

\footnotetext{
${ }^{41}$ Pelzer, « Guillaume de Leus (de Levibus) frère prêcheur de Toulouse », 405 et 409.

${ }^{42}$ Voir à ce propos KAEPPELI, « Guillelmus de Levibus (Leus) Tolosanus », 109, qui cite l'explicit du traité : «Explicit etiam tractatus... a fratre Guillelmo de Liens ordinis fratrum predicatorum et lectore eorumdem fratrum in convento tholosano ». Voir aussi E. BAYERRI Bertomeu, Los Códices Medievales de la Catedral de Tortosa, Barcelona 1962, 381.

${ }^{43}$ Guillaume fait référence à sept reprises à son commentaire des Sentences (livres I-III), cinq fois dans son commentaire au Livre des Causes (Cod. Borgh. 352, ff. 50r, 59v, 77v, 87r et $87 \mathrm{v}$ ) et deux fois dans son commentaire aux Décrétales (Cod. Borgh. 165, ff. 75r et 12r). Voir à ce propos Pelzer, "Guillaume de Leus (de Levibus) frère prêcheur de Toulouse ", 404-5. Le répertoire de F. STEGMÜLleR, Repertorium commentatorium in Sententias Petri Lombardi, Würzburg 1947, ainsi que son supplément réalisé par V. DOUCET, Commentaire sur les Sentences, Quaracchi 1954, ne mentionnent pas cette lecture de Guillaume de Leus (ce qui s'explique par le manque de support matériel du commentaire).

${ }^{44}$ PIRON, «Les studia franciscains de Provence et d'Aquitaine (1275-1335) », 344 ; voir les sources qu'il cite concernant cet exercice toulousain, entre autres concernant Guiral Ot. Voir aussi PELzER, « Guillaume de Leus (de Levibus) frère prêcheur de Toulouse », 397-98. Cf. BAV, Cod. Borgh. 156, f. 1r: « ego frater Guillelmus de Lieus, nacione tholosanus, ordinis fratrum predicatorum, et Tholose lector fratrum prefati ordinis ", cité par PELzER, « Guillaume de Leus (de Levibus) frère prêcheur de Toulouse », 406.
} 
logique. Cet exercice universitaire est habituellement réalisé par un lecteur en théologie appartenant à un ordre mendiant, à la demande de la faculté de droit. Les lectures proposées par Guillaume de Leus aux étudiants toulousains concernent six Décrétales: Firmiter (Décrétales de Grégoire IX, I, 1, c. 1 ; sur la Trinité), Damnamus (Décrétales de Grégoire IX, I, 1, c.2 ; sur les erreurs de Joachim de Flore et d'Amaury de Bène) et Fideli ac devota professione (Sexte de Boniface VIII, I, 1, c.1 ; sur la procession du Saint Esprit) ; puis Cum Marthe, De homine et In quadam nostra (Décrétales de Grégoire IX, III, 41, cc.6-8 ; sur la messe). Il n'est pas étonnant non plus que Guillaume de Leus propose à la lecture du cardinal de la Chapelle, ancien professeur de droit civil à l'Université d'Orléans, ces enseignements "universitaires" qu'il a donnés à la faculté, comme il l'affirme lui-même dans l'explicit ${ }^{45}$.

Quant au commentaire du Livre des Causes, l'explication est moins assurée. Dans quel contexte a-t-il eu l'occasion de composer - ou peut-être même de lire ${ }^{46}$ - ce commentaire ?

Il semble, tout d'abord, que le Livre des Causes ait été commenté dans les couvents dominicains, plus précisément dans les studia naturarum ${ }^{47}$ écoles où les jeunes religieux étudient durant deux ans la philosophie, de fin septembre à fin juin ${ }^{48}$ - et, à partir de 1327-1330 et de l'ajout d'une troisième année d'étude ${ }^{49}$, durant l'enseignement de la philosophie morale (studium moralis philosophie). Il est vrai que les textes que nous possédons sur ce sujet, même s'ils concernent précisément les couvents de la Province de Toulouse, sont plus tardifs, à savoir 1330 ; ils témoignent cependant de la mise en place d'un cours biennal de philosophie morale, avec deux cy-

\footnotetext{
${ }^{45}$ BAV, Cod. Borgh. 156, f. 35v : « Explicit totalis exposicio iustius decretalis necnon et omnium sex decretalium, quas per ordinem exposui, sicut eas in hoc anno in scolis iuristarum Domino largiente <exposui> », cité par PELZER, " Guillaume de Leus (de Levibus) frère prêcheur de Toulouse », 409.

${ }^{46}$ Il est vrai que le texte comporte un certain nombre de formes d'oralité, tel le très récurrent attende tamen.

${ }^{47}$ À la fin du XIII ${ }^{\mathrm{e}}$ siècle, le jeune religieux dominicain, après deux ou trois ans de probation dans un couvent, doit suivre tout d'abord deux ou trois ans de formation en logique dans un studium arcium, puis deux ou trois ans de formation en philosophie naturelle et morale dans un studium naturarum, avant de débuter ses trois ans d'études en théologie. Voir à ce propos DouAIs, Essai, 58, et A. MAIERÙ, University Training in Medieval Europe, trans. D.N. PRYDS (Education and Society in the Middle Ages and Renaissance 3), LeidenNew York 1994, 7-8.

${ }^{48}$ DouAis, Essai, 62.

${ }^{49}$ DouAis, Essai, 70-72.
} 
cles de leçon par année : durant la première, un cours principal fait lire l'Éthique à Nicomaque, et un cours secondaire les Magna moralia, l'Économique et le Livre des Causes; durant la deuxième année, la Politique est étudiée dans le cours principal et la Rhétorique dans le secondaire ${ }^{50}$. Or, Guillaume de Leus, en tant que sous-lecteur et lecteur de théologie dans plusieurs couvents de la première province de Provence, puis de Toulouse, enseigne évidemment les cours principaux de théologie (Bible et Sentences ainsi que théologie morale et droit canon), mais a aussi pu y enseigner la logique et la philosophie ${ }^{51}$. D'ailleurs, d'autres lectores en théologie - dont les deux grands maîtres Albert le Grand et Thomas d'Aquin - ont avant lui commenté le Liber de causis ${ }^{52}$.

De plus, la forme que prend l'Exposicio de Guillaume de Leus, à savoir - comme nous le verrons plus bas ${ }^{53}$ - l'alternance du commentaire suivi avec divisio textus et de questiones, colle tout à fait aux exercices scolaires réalisés dans les studia dominicains ${ }^{54}$. Le lecteur, en effet, donne, d'un côté, des leçons sur les textes d'autorité et, de l'autre, organise des disputes différentes bien sûr de celles solennelles de quodlibet, tenues par les maîtres en Avent et en Carême - qui consistent à faire participer les élèves les plus avancés : le lecteur énonce une thèse à partir d'un matériel choisi (par exemple un ouvrage), qu'un répondant soutient, et contre laquelle un oppo-

\footnotetext{
${ }^{50}$ Voir DouAIs, Essai, 72, qui cite les Actes du chapitre de Montauban de 1330 : « Assignamus Studia moralis philosophie : primum ponimus in Carcassona, lectorem fr. A. de Monassuto; secundum assignamus in Caturco, lectorem fr. P. de Maricalmo. Volumus autem et ordinamus quod dicti magistri, anno isto, legant pro principali leccione librum Ethicorum, pro secunda vero Magna Moralia, Ychonomicam, et librum de Causis. Sequenti anno vero, legant pro principali leccione librum Politicorum, pro secunda vero libri Rhetoricorum ». Cf. aussi A. MAIERÙ, «Formazione culturale e techniche d'insegnamento nelle scuole degli Ordini mendicanti », dans Studio e studia : le scuole degli Ordini mendicanti tra XIII e XIV secolo. Atti del XXIX Convegno internazionale, Assisi, 11-13 ottobre 2001, Spoleto 2002, 3-31, ici 20-21, et IDEM, University Training in Medieval Europe, 12-13 nn. 59-60. Voir aussi M.M. MulchaheY, «First the Bow is Bent in Study....» Dominican Education before 1350, Toronto 1998, 273-74.

${ }^{51}$ Voir MAIERÙ, «Formazione culturale e techniche d'insegnamento nelle scuole degli Ordini mendicanti », 23-24 : «Il lettore insegna teologia, ma può insegnare anche logica e filosofia ». Maierù affirme en outre (University Training in Medieval Europe, 21) que la lecture des Sentences était particulièrement assignée au sous-lecteur des studia, avant que ne se crée la charge spécifique de lector Sentenciarum : «next to the lector or lector principalis, was a sublector or bachelor, who in secunda lectione reads the Sentences ».

52 Outre Albert le Grand et Thomas d'Aquin, DouAIs, Essai, 72-73, parle du commentaire du Liber de causis d'un autre dominicain, frère Tancrède de Bologne.

${ }^{53}$ Voir infra, 312-14.

${ }^{54}$ Voir DouAIS, Essai, 67-68 et 75-80.
} 
sant formule des objections; le répondant résout l'objection par une réponse, que l'opposant tente de contrer, etc. Le lecteur peut alors trancher la question $^{55}$.

Ajoutons enfin que la lecture du Livre des Causes de Guillaume de Leus, comme nous le verrons plus bas ${ }^{56}$, semble très adaptée à un public de jeunes conventuels.

\section{La datation de ses œuvres}

Alors qu'il est lecteur de théologie au couvent de Toulouse, Guillaume de Leus choisit certaines de ses productions pour les dédier au cardinal Pierre de la Chapelle-Taillefer. Comme l'a relevé Sylvain Piron, et comme Guillaume le reconnaît lui-même dans la lettre dédicatoire de son Explication des six Décrétales, le dossier est aussi envoyé à la demande des juristes de l'Université de Toulouse, qui sans doute ont des intérêts à ce que sa promotion au titre de docteur ait lieu, afin de redonner un certain lustre à l'enseignement toulousain :

Ad quod eciam me induxit instancia universitatis iuristarum venerabilis et Deo amabilis studii tholosani ${ }^{57}$.

L'Université de Toulouse, qui a été fondée en 1229 dans le but d'extirper les restes de l'albigéisme et d'instaurer une doctrine orthodoxe ${ }^{58}$, a pratiquement été dépourvue de Faculté de théologie après les régences de Roland de Crémone et Jean de Saint-Gilles, les deux premiers maîtres dominicains de Paris ${ }^{59}$. Seuls ont fonctionné alors les studia des frères mendiants,

\footnotetext{
${ }^{55}$ Voir à ce propos MAIERÙ University Training in Medieval Europe, 26-28 : « for collationes, it was customary to delegate a friar to recite a questio determinata in scholis and to think up objections for him (opponunt recitanti) so that the friars could practice opponendo et respondendo.... Students gradually began the difficult exercise of disputation and they are guided by someone who had the responsability to decide when and on which arguments to give them. The student's master after settling on the subject with the lector..., then found the books which provided the necessary material; he alerted those who were interested at the appropriate time; he gave them the bibliography, and he finally decided who should respond and who could begin to act as opponens ». Voir aussi IDEM, « Formazione culturale e techniche d'insegnamento nelle scuole degli Ordini mendicanti », 26.

${ }^{56}$ Voir à ce propos la présentation de ce point infra, 314 .

${ }^{57}$ BAV, Cod. Borgh. 165, f. 1ra. Voir à ce propos PIRON, «Les studia franciscains de Provence et d'Aquitaine (1275-1335)», 321-22, 325.

${ }^{58}$ Voir à ce propos PIRON, «Les studia franciscains de Provence et d'Aquitaine (12751335) », 321, qui cite C.E. SмITH, The University of Toulouse in the Middle Ages. Its Origins and Growth to 1550 AD, Milwaukee 1958.

${ }^{59}$ Voir Y. Dossat, «L'université de Toulouse », dans Les Universités du Languedoc au
} 
comme des « quasi-faculté[s] de théologie ${ }^{60}$. L'exigence d'une croisade contre le catharisme s'étant estompée, l'Université de Toulouse a en effet cessé d'être une priorité pour l'ordre dominicain. Ce n'est qu'en 1290 qu'Ysarn de Saint-Paul, chancelier de l'Université, demande au chapitre provincial de Pamiers de pourvoir à nouveau d'un maitre dominicain la chaire de théologie ${ }^{61}$. Avec la nomination de Raymond Guilha en 1295, la voix du Chancelier semble avoir été entendue ${ }^{62}$. Elle le sera à nouveau en 1311, d'une certaine manière, avec la promotion de Guillaume de Leus au rang de maître et son retour au couvent de Toulouse ${ }^{63}$.

Les œuvres dédiées à Pierre de la Chapelle, du moins celles qui nous sont parvenues dans les deux manuscrits Borghèse - car il se peut que d'autres œuvres, tel le commentaire des Sentences, aient aussi été offertes au cardinal -, sont, comme nous l'avons déjà mentionné, l'Explication $d u$ Livre des Causes (Cod. Borgh. 352) et l'Explication des six Décrétales $\left(\right.$ Cod. Borgh. 156) ${ }^{64}$.

Il importe avant tout pour nous de définir la datation de l'Exposicio in Librum de causis. Concernant le terminus post quem, la seule limite pertinente se rapporte à la rédaction du Super Librum de causis rédigé par Gilles de Rome, que Guillaume reprend à de très nombreuses reprises, daté de $1289-1291^{65}$. Le commentaire du Livre des Causes de Guillaume a ainsi pu être élaboré bien avant 1303 et son lectorat en théologie au couvent de Toulouse, durant la première partie de sa carrière dans les différents couvents provençaux (Carcassone, Narbonne, Montpellier, Toulouse) et suite à ses études parisiennes ${ }^{66}$.

XIII siècle (Les Cahiers de Fanjeaux 5), Toulouse 1970, 58-91, ici 68-69.

${ }^{60}$ S. PIRON, « Les studia franciscains de Provence et d'Aquitaine (1275-1335) », 322.

${ }^{61}$ Cf. IdEm, Ibid. Voir aussi E. Deluaruell, «L'université de Toulouse à la fin du XIII ${ }^{\mathrm{e}}$ siècle », dans Les Universités du Languedoc au XIII siècle, 108-15, ici 114, et DoUAIS, Essai, 148-49, où la réponse de Bernard de Trilia, prieur provincial, à Ysarn est reproduite.

${ }^{62}$ Voir supra, 301 n. 28.

${ }^{63}$ Guillaume de Leus est maître en théologie au couvent dominicain, mais son retour à Toulouse a un impact qui se ressent sur l'intégralité de la communauté "scientifique » toulousaine.

${ }^{64}$ Associée à une Explication de la messe et une Histoire de la Passion du Christ, comme illustration des trois dernières décrétales.

${ }^{65}$ Voir à ce propos infra, 315-16. Pour la datation du commentaire, voir S. DonATI, « Studi per una cronologia delle opere di Egidio Romano ", dans Documenti e studi sulla tradizione filosofica medievale 1 (1990), 1-111, 65-71.

${ }^{66}$ Voir la discussion supra, 305-7. Ceci est possible car, dans l'épître dédicatoire au commentaire du Livre des Causes, Guillaume de Leus ne mentionne pas qu'il est lecteur au 
Concernant le terminus ante quem, une alternative se présente : soit le commentaire a été rédigé et dédié après que Guillaume est devenu maître, et dans ce cas il coïncide avec sa nomination à l'épiscopat (1313), soit le commentaire a été rédigé durant la carrière de Guillaume dans les couvents avant sa promotion au rang de maître, et le terminus ante quem est alors 1309.

Si l'on suit la piste de la deuxième option, qui semble plus probable, les informations que transmet l'autre commentaire sont alors capitales pour corroborer le terminus ante quem de l'Explication du Livre des Causes. Or, l'on sait que l'Explication des Décrétales a été réalisée alors que le dominicain est lecteur au couvent de Toulouse, à savoir entre 1303 et 1309, et qu'il l'a dédiée après 1305 , date de la nomination au cardinalat de Pierre de la Chapelle ${ }^{67}$. Il est ainsi certain que la dédicace (du commentaire aux Décrétales) de Guillaume au cardinal a précédé l'obtention de la licencia docendi, annoncée et réalisée d'ailleurs par l'intermédiaire de ce dernier, et l'on peut imaginer que ces liens entre Guillaume et Pierre de la Chapelle ont contribué à sa promotion ${ }^{68}$. L'hypothèse d'une dédicace antérieure au nouveau statut de magister theologie semble d'autant plus probante que c'est les juristes de l'Université de Toulouse qui encouragent le dominicain à offrir son œuvre au cardinal, afin de promouvoir les études toulousaines et accueillir un nouveau maître dans la communauté « scientifique ». Ainsi, si l'on envisage les cas des deux commentaires - Décrétales et Livre des Causes - comme symétriques, l'on peut proposer la date vraisemblable de 1309 comme terminus ante quem.

couvent de Toulouse, ce qu'il fait dans l'autre épître. Cet extrait est reproduit infra, dans la n. 67. Voir l'intégralité de l'épître dans l'Annexe 2.

${ }^{67}$ Pour le lectorat de Guillaume, voir supra, 304 n. 44. Guillaume de Leus donne ce titre à Pierre de la Chapelle-Taillefer dans les épîtres dédicatoires, par exemple: BAV, Cod. Borgh. 352, f. 1ra : « ...preclaro domino Petro de Capella... sacrosancte et romane Ecclesie venerabili cardinali devotus sibi in Christo frater Guillelmus de Liues, ordinis [ordinum V] fratrum predicatorum, nacione Tolosanus, fructum aliqualem laboris sui studii sibi offerens ». Or Pierre est nommé cardinal par Clément V en décembre 1305 (cf. U. CHEVALIER, Répertoire des sources historiques du Moyen Âge, Bio-bibliographie 2, Paris 1907, col. 3702).

${ }^{68}$ C'est ce qu'a soutenu Pelzer, « Guillaume de Leus (de Levibus) frère prêcheur de Toulouse », 396, et, après lui, tous les auteurs qui se sont intéressés à Guillaume de Leus. Il existe d'ailleurs d'autres religieux qui ont dédié des œuvres à des personnages importants afin d'obtenir un titre de magister theologie, tel le cas - mentionné par Bill Duba, à partir du ms. Borghèse 329 - de Pierre Auriol, qui offre, en 1317, son commentaire au premier livre des Sentences à Jean XXII, ce qui garantit sa promotion à la licencia docendi l'année suivante. 
Chronologie des œuvres de Guillaume de Leus
(1) $1283-1308$
Commentarius in Libros I-III Sentenciarum
(2) $1289-1309(?)$
Exposicio in Librum de causis
(3) $1303-1309$
Exposicio sex Decretalium
(4) $1303-1309$
Exposicio Misse
(5) $1303-1309$
Historia Passionis Christi
(6) 1303-1309
Tractatus de predestinacione electorum et reproba- cione malorum

Les justifications des datations sont les suivantes :

Pour (1), Guillaume de Leus enseigne pour la première fois la théologie en 1283 et a déjà rédigé ce commentaire quand il compose (2) et (3).

Pour (2), Guillaume cite le commentaire au Livre des Causes de Gilles de Rome (ap. 1289), et, si l'on fait l'hypothèse que (2) a été dédié au cardinal avant ou au même moment que (3), (4) et (5), on peut proposer 1309 comme date limite, d'autant plus si l'hypothèse des dédicaces au cardinal, précédant et favorisant sa nomination au rang des maîtres, se révèle exacte.

1305 représente le terminus post quem non de la composition, mais de la dédicace, puisque c'est la date où Pierre de la Chapelle devient cardinal, titre que lui donne Guillaume dans les lettres dédicatoires (c'est le même cas pour 3,4 et 5 ).

Pour (3), Guillaume est lecteur du couvent de Toulouse quand il fait cet exercice et quand il dédie son œuvre au cardinal, or il est lecteur entre 1303 et 1309 .

Pour (4) et (5), les dates sont les mêmes que (3), car les traités sont écrits pour développer certains points présentés dans (3) et ils sont dédiés avec (3) au cardinal.

Pour (6), l'explicit affirme que Guillaume est lecteur du couvent de Toulouse, ce qui est le cas entre 1303 et 1309.

\section{Le manuscrit Vatican, Cod. Borgh. 352}

L'unique manuscrit connu aujourd'hui contenant le commentaire de Guillaume de Leus au Liber de causis est le codex de la Bibliotheca Apostolica Vaticana, Cod. Borgh. 352. En parchemin, écrit sur deux colonnes, il se compose de 87 folios qui ne contiennent que ce commentaire. Selon la description qu'en propose Anneliese Maier ${ }^{69}$, le tout semble avoir été écrit au

${ }^{69}$ A. MAIER, Codices Burghesiani Bibliothecae Vaticanae (Studi e testi 170), Città del 
début du XIV ${ }^{\mathrm{e}}$ siècle d'une seule main, les grandes lettres et les signes de paragraphes sont de couleur rouge et bleu (ou violet). Dans les marges, outre les numéros des propositions, on trouve à quelques endroits des corrections et des remarques soit au crayon, soit à l'encre. Les fascicules sont presque tous des seniones, les folios sont numérotés au sommet des pages de droite et la couverture est en parchemin.

Ce manuscrit provient de la bibliothèque des papes d'Avignon ${ }^{70}$, puisqu'il apparaît dans les inventaires : celui de 1369 , sous le numéro $445,{ }^{71}$ commandé par Urbain V; celui de 1375, avant le retour de Grégoire XI à Rome, sous le numéro $457^{72}$; celui de 1407, premier catalogue d'Avignon, sous le numéro $1514^{73}$; celui de 1411 , qui décrit les volumes restés à Avignon après le départ de Benoît XIII et de ses livres pour Peñiscola, sous le numéro $622^{74}$; enfin, celui de 1594 , commandé par Clément VIII, sous le chiffre $146^{75}$. La bibliothèque papale d'Avignon, qui s'est développée au $\mathrm{XIV}^{\mathrm{e}}$ siècle à partir de rien, avant de devenir la collection la plus riche de son temps, s'est évanouie à peu près aussi rapidement qu'elle était née. La moitié des quelque 2000 volumes est en effet emportée par Petrus de Luna, l'antipape Benoît XIII, lorsqu'il quitte Avignon. Le reste y demeure jusqu'au début du XVII ${ }^{\mathrm{e}}$ siècle (1604), avant de passer en la possession de la famille Borghèse, à Rome, et ce jusqu'à ce qu'en 1891, Franz Ehrle en fasse l'acquisition pour la Bibliothèque Vaticane ${ }^{76}$. C'est l'itinéraire qu'a

\footnotetext{
Vaticano 1952, 402-3.

${ }^{70}$ Le manuscrit ne semble cependant pas avoir été transmis à la bibliothèque suite aux dépouilles du cardinal Pierre de la Chapelle-Taillefer, selon les inventaires de M.-H. JULLIEN DE Pommerol et J. Monfrin, Bibliothèques ecclésiastiques au temps de la papauté d'Avignon II : Inventaires de prélats et de clercs français, Paris 2001.

${ }^{71}$ F. EHRLE, « Recensio librorum anno 1369 in palatio Avenionensi asservatorum », dans Historia bibliothecae Romanorum pontificium tum Bonfitianae tum Avenionensis, $324 \mathrm{n}^{\circ}$ 445. Inventaire réalisé sous l'autorité du cardinal Philippe de Cabassole.

${ }^{72}$ F. EHRLE, « Recensio Bibliothecae Avenionensis a Gregorio XI concinnata ac disposita », dans Historia bibliothecae Romanorum pontificium tum Bonfitianae tum Avenionensis, 489 : « Item in volumine signato per LIIII scriptum G. de Hionis, super libros de causis ».

${ }^{73}$ Cf. M.-H. Jullien de Pommerol et J. Monfrin, La bibliothèque pontificale à Avignon et à Peñiscola 2, Rome 1991, 964.

${ }^{74}$ MAIER, « Der Katalog der päpstlichen Bibliothek in Avignon vom Jahr 1411 », $154 \mathrm{n}^{\circ}$ 622 : «Item Scriptum fratris Guillelmi de Lieus super librum de causis cop. Pelle viridi et inc. In $2^{\circ}$ folio cause secunde et finit in penultimo celestes $c i »$.

${ }^{75}$ MAIER, « Der letze Katalog der päpstlichen Bibliothek von Avignon (1594)», $224 n^{\circ}$ 146 : «Guillermi de Lives de causis ». Inventaire réalisé sous l'autorité du cardinal-légat Ottavio Acquaviva.

${ }^{76}$ Voir à ce propos MAIER, « Der Katalog der päpstlichen Bibliothek in Avignon vom Jahr
} 
suivi le manuscrit de Guillaume de Leus ${ }^{77}$.

\section{Le commentaire au Liber de causis}

\section{Le genre littéraire}

L'épître dédicatoire de Guillaume de Leus au cardinal Pierre de la Chapelle est suivie d'une exposition de la méthode utilisée pour commenter le Livre des Causes $^{78}$. Le dominicain reproduira tout d'abord la proposicio du Liber de causis en grandes lettres, puis son commentum, à savoir, respectivement, la première phrase puis la suite du texte des propositions ${ }^{79}$. Guillaume explique qu'il analysera ensuite cette proposicio et son commentum en présentant une division du texte (cum propriis divisionibus) et en rendant attentif aux nombreux éléments intéressants (multis necessariis notabilibus). Il y rattachera (adnecto), enfin, une question à propos des points les plus difficiles et utiles apparaissant dans la proposition.

Guillaume distingue en effet, dans chaque proposition, ce qu'il nomme proprement la proposicio ou le théorème initial du Liber de causis, que le manuscrit transcrit en caractères plus grands, et ensuite le commentum ou sa démonstration, qui comprend le reste du texte. Après avoir transcrit le texte de chaque proposition, c'est-à-dire de la proposicio et du commentum, Guillaume l'explique d'abord par un commentaire analytique et suivi (organisé selon la divisio textus), puis par une question qu'introduit le mot queritur. Ainsi, chaque proposition commentée l'est doublement: premièrement, par une analyse textuelle serrée qui met en lumière les raisonnements successifs et leur enchaînement, ainsi que les problèmes soulevés par l'argumentation (souvent introduits par l'expression attende) ; deuxièmement, par une question, choisie par l'auteur en fonction des thématiques abordées et traitée à la manière des questiones disputate de la Faculté des $\mathrm{Arts}^{80}$, à savoir libellé de la question; et videtur quod; arguments pro; in

$1411 »$.

${ }^{77}$ Nous n'avons pas présenté d'arguments liés à la description des manuscrits (par exemple, pour servir à la datation du commentaire), car le ms. Borgh. 352 semble être trop fautif pour être celui dédié au cardinal.

${ }^{78}$ Voir le texte dans l'Annexe 2.

${ }^{79}$ Relevons en outre que le copiste du manuscrit souligne l'ensemble du texte tiré du $\mathrm{Li}$ ber de causis, et réitère ce «style" chaque fois qu'un passage du De causis est cité par Guillaume.

${ }^{80}$ Voir à ce propos O. WeIJERS, Queritur utrum : recherches sur la disputatio dans les universités médiévales (Studia Artistarum 20), Turnhout 2009. Selon les différents types de questions décrits par cette même chercheur dans son article, «L'enseignement du trivium à 
contrarium; determinacio questionis; réponses aux arguments pro.

Cette manière d'étudier chaque proposition du Liber de causis, tant par le commentaire linéaire et la divisio textus que par une questio est spécifique à Guillaume de Leus. Les premiers commentaires d'avant 1260, tels celui de Roger Bacon et celui attribué à Henri de Gand, ainsi que celui plus tardif de Siger (1274-1276), privilégient l'analyse par questions, en posant plusieurs questions à chaque proposition. Il est vrai que parmi ces premiers commentaires, celui du ps.-Adam de Bocfeld associe la lecture suivie et les questions, mais de manière irrégulière: huit propositions n'ont que le commentaire littéral, d'autres n'ont que les questions, qui sont souvent multiples pour une même proposition ${ }^{81}$. Les autres commentaires antérieurs, tels le De Causis et processu universitatis a Prima causa d'Albert le Grand, le Super Librum de causis expositio de Thomas d'Aquin et le Super Librum de causis de Gilles de Rome procèdent, quant à eux, respectivement sous la forme d'une paraphrase (pour ce qui concerne le second livre d'Albert), de l'exposicio de l'ensemble de chaque proposition ${ }^{82}$, et du commentum des différentes parties de la proposition. Parmi les commentaires inédits dont s'occupe l'équipe de Cluj $^{83}$, l'on trouve soit des commentaires par lemmes (par exemple Ausburg, $\mathrm{SSB}, 4^{\circ}$ Cod 68 ), soit des commentaires par questions (par exemple Erfurt, UB, Dep. Erf., CA Q.316; Wien, ÖNB, Cod. 2330 ; Paris, BNF, Ms. lat. 15819). Seul parmi ceux connus aujourd'hui, le ms. Worcester, Cathedral and Chapter Library, Ms. Q.90 semble mêler le commentaire linéaire et les questions, mais de manière bancale.

Guillaume de Leus, de son côté, couple les deux méthodes d'analyse et

la Faculté des Arts de Paris : la questio », dans Manuels, programmes de cours et techniques d'enseignement dans les Universités médiévales, éd. J. HAMESSE (Textes, études, congrès 16), Louvain-la-Neuve 1994, 57-74, 73, on se rapprocherait ici des « questions constituant à elles seules un commentaire (elles se rapportent au contenu du texte ou du moins à son sujet ; elles sont dipsutées selon la méthode dialectique) », bien que justement elles ne soient pas un commentaire à elles seules, puisqu'elles sont couplées à une exposicio.

${ }^{81}$ Voir à ce propos C. D’AnConA, «Philosophus in Libro de Causis. Le Liber de Causis comme ouvrage aristotélicien dans les commentaires de Roger Bacon, du Ps. Henri de Gand et du Ps. Adam de Bocfeld ", dans IDEM, Recherches sur le Liber de Causis, Paris 1995, 195-228, 206-7.

${ }^{82}$ Pour la qualification précise de l'interprétation de ces deux auteurs, voir A. DE LIBERA, " Albert le Grand et Thomas d'Aquin interprètes du Liber de Causis », dans Revue des sciences philosophiques et théologiques 74 (1990), 347-78.

${ }^{83}$ Voir à propos des commentaires contenus dans le mansucrit Ausburg, SSB, $4^{\circ}$ Cod 68, l'article d'Alexandra Baneu et Dragos Calma dans ce numéro. Le manuscrit Erfurt, UB, Dep. Erf., CA Q.316 est étudié actuellement par Iulia Székely, alors que Mihai Maga étudie les manuscrits Wien, ÖNB, Cod. 2330, et Paris, BNF, Ms. lat. 15819. 
d'investigation de manière structurée : pour la première démarche, il réalise un travail très complet, compilant différentes sources ; pour la seconde, il diffère de ses prédécesseurs en se concentrant sur une unique interrogation pour chaque proposition (sauf pour la proposition 21 qui produit deux questions).

Relevons, enfin, qu'en comparaison aux autres commentaires inédits, l'interprétation proposée par Guillaume de Leus semble être particulièrement théologique, au sens chrétien du terme - et non seulement aristotélicien -, et ainsi principalement destinée à un public de jeunes étudiants des studia conventuels. Cela se déduit du libellé des questions, tant des intérêts qu'elles dévoilent - unicité divine (q.1 ${ }^{84}$ ), primauté de l'être (q.2), création du monde (q.4), connaissance des anges (q.8), immédiateté de l'action divine (q.16), simplicité divine (q.18), gratuité de la grâce (q.21b) - que du vocabulaire utilisé - Deus (q.16, q.18, q.21a et b, q.24) plutôt qu'à chaque fois causa prima; angelus (qq.7-8, 10) plutôt que constamment intelligen$\mathrm{cia}^{85}$, etc. - ainsi que des sources utilisées - outre Aristote, principalement la Bible, Augustin et le pseudo-Denys ${ }^{86}$.

Cette lecture théologique ressort aussi des distinctions qu'il propose régulièrement entre le point de vue des philosophes et celui des théologiens ${ }^{87}-$ ce qui l'amène à relever l'error Commentatoris concernant l'unité de l'Intellect ${ }^{88}$ - et de l'utilisation de formules telles que in contrarium est fides nostra, nos instructi per fidem nobis traditam in scriptura sacra ou encore hoc enim derogat catholice fidei ${ }^{89}$, lorsqu'il veut se distancer de certaines positions du Liber de causis, ou du moins d'une lecture platonicienne de ces thèses.

\footnotetext{
${ }^{84}$ Pour le libellé des questions, voir infra, Annexe 1, 325-27.

${ }^{85}$ Voir à ce propos l'article de S. PIRON, « Deplatonising the Celestial Hierarchy. Peter John Olivi's Interpretation of the Pseudo-Dionysius ", dans Angels in Medieval Philosophy Inquiry. Their Function and Significance, ed. I. IRIBARREN and M. LENZ, Aldershot 2008, 29-44, qui présente les différentes stratégies de théologiens (Albert, Thomas, Olivi) concernant les relations entre les anges de la tradition chrétienne et les substances intellectuelles de la tradition philosophique, en particulier néo-platonicienne. Il semblerait - simplement déjà en notant l'hésitation concernant le vocabulaire - que la voie de Guillaume, contrairement à celles d'Albert et d'Olivi, permette une identification partielle - lorsque cela ne va pas à l'encontre des vérités de la foi - entre les anges et les intelligences.

${ }^{86}$ Voir infra, 318-19, Les sources explicites, tableaux 2, 3 et 4.

${ }^{87}$ Voir par exemple BAV, Cod. Borgh. 352, q.3, f. 8rb : « fuerunt due opiniones, philosophorum et theologorum »; q.14, f. 34ra : « huic sentencie philosophorum concordat sentencia theologorum »; q.23, f. 62ra : « que a philosophis etiam denominantur anime celorum et a theologis denominantur angeli ». Voir aussi q.4, f. 11ra-b ; q.5, ff. 12vb, 13va, 14ra.

${ }^{88}$ Cf. BAV, Cod. Borgh. 352, q.4, f. 10vb.

${ }^{89}$ Voir respectivement BAV, Cod. Borgh. 352, q.1, f. 3vb ; q.5, f. 14rb ; q.10, f. 26ra.
} 


\section{Les propositions commentées}

Des trente-deux propositions que comporte la traduction latine du Liber de causis, le manuscrit Borgh. 352 ne reproduit et n'explique que les propositions 1 à 30 , selon une version quelque peu différente du texte de l'édition de Pattin ${ }^{90}$, mais sans grandes modifications.

Il est cependant possible que l'unique exemplaire que nous possédons de ce commentaire, à savoir le manuscrit Cod. Borgh. 352, ne soit pas complet relativement à l'original puisque Guillaume de Leus relève par deux fois - au début de l'explication de la première proposition et dans l'explicit - que le Liber de causis possèdent trente-deux propositions, sans préciser qu'il ne les reproduira pas toutes, et affirmant même qu'il a proposé l'exposition de l'intégralité de l'écrit :

Hinc autem in libro quo XXXII proposiciones cum commentis suis continentur, premittit actor hanc primam proposicionem tractantem de primariis causis, quasi quoddam prohemium respectu tocius operis.

Explicit igitur Liber de causis, divisus per theoremata, continens in se XXXII proposiciones cum totidem commentis iuxta numerum dictarum proposicionum..., quas [proposiciones] ego cum auxilio et gracia Dei cum propriis commentis exposui, apponens singulis proposicionibus et commentis singulas et proprias questiones iuxta numerum et materiam et exigenciam dictarum proposicionum.... Sicut incepi ex confidencia Christi, sic eciam auxiliante eius gracia feliciter consummavi ad $|87 \mathrm{vb}|$ laudem et gloriam eius, cui est honor et imperium quasi cause primarie et universali cum Patre et Spiritu Sancto in secula seculorum. Amen. Explicit scriptum et exposicio tocius Libri de causis ${ }^{91}$.

Guillaume le dominicain, exprime ici, avec l'humilité de mise, la nouveauté de son commentaire : il a posé à chaque proposition et son commentaire une question propre et singulière, selon le nombre, la matière et l'exigence de celle-là.

Les sources : Rapprochements textuels avec les commentaires de Gilles de Rome, Thomas d'Aquin et Albert le Grand

Selon une première enquête effectuée avant tout à partir du prologue, de l'exposition de la première proposition, ainsi que des sources explicites de l'ensemble du commentaire, il semblerait que Guillaume de Leus s'inspire

${ }^{90}$ Le Liber de causis, éd. A. PATTIN, dans Tidschrift voor Filosofie 28 (1966), 90-203, avec les propositions de corrections de R. C. TAYLOR, « The Liber de causis : A Preliminary List of Extant MSS », dans Bulletin de philosophie médiévale 25 (1983), 63-84.

${ }^{91}$ BAV, Cod. Borgh. 352, ff. 1vb et $87 \mathrm{va}-\mathrm{b}$. 
largement du Super Librum de causis de Gilles de Rome. Cela se vérifie en particulier dans la structure du prologue ${ }^{92}$, les autorités citées et le contenu du commentaire suivi du Liber de causis, que le dominicain associe à une division du texte, tout comme l'avait fait le doctor fundatissimus. En effet, leur divisio textus est quasi identique et les différentes parties du raisonnement sont caractérisées de la même manière - tel argument est le corollaire (corelarium) de cet autre, ou son support (fulcimentum $)^{93}$.

Quant au commentaire de Thomas d'Aquin, il est utilisé par Guillaume, tout d'abord dans le prologue, quand il s'agit de discuter du rapport entre l'Elementacio theologica et le Liber de causis ${ }^{94}$, puis dans l'étude des propositions, tant pour enrichir sa réflexion dans le commentaire suivi - qui, dans ce cas, ajoute quelque chose à l'analyse de Gilles de Rome - que pour étayer ses démonstrations dans les réponses aux questions, que ce soit à travers un élément de preuve ou la citation d'une autorité, empruntés à Thomas $^{95}$. Ceci se manifeste particulièrement par l'utilisation importante que Guillaume fait du pseudo-Denys, aux côtés de Proclus et des thèses platoniciennes ${ }^{96}$, pour se positionner, la plupart du temps, face à Proclus et aux côtés de Denys - en particulier dans son refus des formes séparées et des hypostases divines ${ }^{97}$.

Le commentaire albertinien, présentation de la théologie aristotélicienne selon la ligne interprétative arabe ${ }^{98}$, n'est pas absent du commentaire de Guillaume, si l'on observe la nature des sources explicites relevées ${ }^{99}$. Il a inspiré, de plus, différents éléments du prologue concernant l'auteur, la forme et le titre du Livre des Causes : son allusion à la possible " autorité » de David le Juif, la caractérisation de son modus agendi par theoremata, le quadruple titre de cette œuvre ${ }^{100}$. En outre, l'axiome opus nature est opus intelligencie, que Guillaume cite tout au début de son prologue pour intro-

\footnotetext{
${ }^{92}$ Voir infra, 321-25.

${ }^{93}$ Cf. BAV, Cod. Borgh. 352, ff. 2ra, 3ra.

${ }^{94}$ Voir infra, 322-23.

${ }^{95}$ Ces affirmations recevront une illustration lors d'une prochaine publication, où nous présenterons l'édition critique du commentaire de Guillaume de Leus à la première proposition du Livre des Causes.

${ }^{96}$ Voir infra, 319-20, Les sources explicites, tableaux 4, 5 et 6.

${ }^{97}$ Voir à ce propos la Préface de l'éditeur du Super Librum De causis Expositio de Thomas d'Aquin, ed. H.-D. SAFFreY, Fribourg (Suisse)-Louvain 1954, XXXVI-XXXVII.

${ }^{98}$ Voir à ce propos DE LIBERA, « Albert le Grand et Thomas d'Aquin interprètes du Liber de Causis », 364-77.

${ }^{99}$ Voir à ce propos infra, 318-21, Les sources explicites, tableaux 1, 7, 12, 14, 15.

${ }^{100}$ Voir à ce propos infra, 322-24.
} 
duire la matière du Liber de causis, est certainement d'invention albertinienne ${ }^{101}$ et est d'ailleurs utilisée à deux reprises dans le De Causis et processu universitatis a Prima Causa ${ }^{102}$, afin d'insister sur le caractère intelligible de l'œuvre de la Nature ${ }^{103}$. L'interprétation de Guillaume de Leus, qui s'inspire de la seconde citation d'Albert, insiste particulièrement sur la hiérarchie existant entre la cause seconde universelle (la Nature) et les causes "intellectuelles", séparées ou premières (comme il les nomme), puisqu'il poursuit son prologue en affirmant :

natura habet totam efficaciam suam et virtutem productivam ex influencia intelligencie. Quod quidem oportet propter duo. Primo quia, cum inferius dependeat et recipiat a superiori, et intelligencia sit superior omni corporali natura.... Secundo quia... natura agit propter finem... quia igitur ista agencia corporalia intellectu carencia non possunt cognoscere (cognosce $\mathrm{V}$ ) ordinem nec proporcionem sui ad finem, indigent moveri et dirigi ad fines suos per agens intellectuale ${ }^{104}$.

\section{Les sources explicites}

Les sources explicites, répertoriées dans l'intégralité du commentaire du Livre des Causes de Guillaume de Leus, ont été organisées en tableaux qui regroupent, selon un classement décroissant d'importance, les autorités présentes, soit dans le prologue $(\mathrm{P})$, soit dans les explicaciones $(\mathrm{E})$, soit dans les questiones (Q) des différentes propositions (distinguées par leur rang, de 1 à 30). Nous indiquons encore le lieu précis où se trouvent les références (foliation traditionnelle) ainsi que, tout au début, le livre (en chiffres romains) et/ou le chapitre (en chiffres arabes) de l'œuvre citée, lorsqu'ils sont mentionnés par l'auteur.

${ }^{101}$ C'est du moins la thèse de L. HöDL, «Opus naturae est opus intelligentiae. Ein neuplatonisches Axiom im aristotelischen Verständnis des Albertus Magnus », dans Averroismus im Mittelalter und in der Renaissance, hrsg. v. F. NIEWÖHNER und L. STURLESE, Zürich 1994, 132-48, et de J. WeISHEIPL, « The Axiom Opus naturae est opus intelligentiae and its Origins ", dans Albertus Magnus. Doctor universalis. 1280/1980, hrsg. v. G. MEYER und A. ZimmermanN, Mayence 1980, 441-63. Le dominicain allemand l'aurait cependant sûrement extrapolé du corpus naturel aristotélicien. On trouve d'ailleurs cette sentence dans les Auctoritates Aristotelis publiées par J. HAMESSE, Louvain-Paris 1974, 139 n²82, sous Commentator, Metaphysica, mais il s'agit d'un locus non inventus.

102 Cf. Albertus Magnus, De Causis et processu universitatis a Prima Causa I.2.8 et II.1.2, ed. W. FAUSER, dans Opera omnia XVII.2 [Ed. Colon.], Münster i.W. 1993, 34 et 62.

103 Voir à propos de l'emploi de cet axiome néoplatonicien par Albert le Grand, WeISHEIPL, "The Axiom Opus naturae est opus intelligentiae and its Origins ».

${ }^{104}$ BAV, Cod. Borgh. 352, f. 1rb. 


\begin{tabular}{|c|c|}
\hline \multicolumn{2}{|r|}{ 1. Philosophus -123 occurrences } \\
\hline Sans autre référence & $\begin{array}{l}\mathrm{P}, 1 \mathrm{ra} ; 1 \mathrm{Q}, 4 \mathrm{rb} ; 3 \mathrm{E}, 8 \mathrm{ra} ; 3 \mathrm{Q}, 9 \mathrm{ra} ; 3 \mathrm{Q}, 9 \mathrm{rb} ; 4 \mathrm{E}, 10 \mathrm{vb} ; 5 \mathrm{E}, 13 \mathrm{vb} ; \\
6 \mathrm{E}, 14 \mathrm{va} ; 6 \mathrm{E}, 15 \mathrm{ra} ; 6 \mathrm{Q}, 15 \mathrm{va}(2 \mathrm{x}) ; 6 \mathrm{Q}, 15 \mathrm{vb}(2 \mathrm{x}) ; 6 \mathrm{Q}, 16 \mathrm{va} ; 7 \mathrm{Q}, \\
18 \mathrm{va}(2 \mathrm{x}) ; 8 \mathrm{Q}, 21 \mathrm{ra}(2 \mathrm{x}) ; 9 \mathrm{E}, 23 \mathrm{ra} ; 11 \mathrm{Q}, 27 \mathrm{rb}(2 \mathrm{x}) ; 11 \mathrm{Q}, 28 \mathrm{rb} ; \\
12 \mathrm{E}, 28 \mathrm{vb} ; 13 \mathrm{E}, 30 \mathrm{va}(2 \mathrm{x}) ; 13 \mathrm{Q}, 31 \mathrm{vb}(2 \mathrm{x}) ; 14 \mathrm{Q}, 34 \mathrm{va}(2 \mathrm{x}) ; 15 \mathrm{E}, \\
35 \mathrm{rb} ; 15 \mathrm{Q}, 37 \mathrm{ra} ; 15 \mathrm{Q}, 37 \mathrm{rb} ; 15 \mathrm{Q}, 37 \mathrm{va} ; 17 \mathrm{E}, 42 \mathrm{vb} ; 18 \mathrm{E}, 45 \mathrm{rb} ; \\
19 \mathrm{Q}, 48 \mathrm{vb} ; 19 \mathrm{Q}, 49 \mathrm{ra} ; 21 \mathrm{E}, 54 \mathrm{rb} ; 21 \mathrm{E}, 55 \mathrm{va} ; 21 \mathrm{aQ}, 55 \mathrm{vb} ; 21 \mathrm{aQ}, \\
56 \mathrm{ra} ; 22 \mathrm{E}, 56 \mathrm{vb} ; 22 \mathrm{E}, 57 \mathrm{ra} ; 22 \mathrm{E}, 58 \mathrm{ra} ; 22 \mathrm{E}, 59 \mathrm{rb} ; 23 \mathrm{E}, 61 \mathrm{va} ; \\
24 \mathrm{Q}, 67 \mathrm{rb} ; 25 \mathrm{Q}, 70 \mathrm{va} ; 26 \mathrm{Q}, 75 \mathrm{vb} ; 26 \mathrm{Q}, 77 \mathrm{vb} ; 28 \mathrm{Q}, 80 \mathrm{ra} ; 29 \mathrm{Q}, \\
83 \mathrm{ra}\end{array}$ \\
\hline Topica & III, 21E, 54vb ; 29E, 82ra \\
\hline Analytica posteriorum & $\mathrm{I}, 1 \mathrm{E}, 2 \mathrm{rb}$ \\
\hline De generacione & II, in fine, 9E, 22rb ; II, circa finem, 24E, 66ra ; I, 29E, 82va \\
\hline De sensu & $15 \mathrm{Q}, 37 \mathrm{ra}$ \\
\hline De celo et mundo & 3Q, 8ra ; 5Q, 13vb; I, 9E, 23ra \\
\hline De anima & $\begin{array}{l}3 \mathrm{E}, 7 \mathrm{va} \text {; III, 6Q, 15va ; III, 8Q, 20va ; III, 8Q, 21 ra ; III, 10E, 25rb ; } \\
\text { I, 10Q, 26ra ; III, 13E, 30ra ; III, 13E, 30rb ; III, 15E, 35ra ; 18E, } \\
\text { 45rb ; III, 19E, 47va ; III, 21 aQ, 55va ; III, 23E, 61vb ; II, 24E, 66ra ; } \\
\text { II, 25E, 68vb ; II, 26E, 72rb ; I, circa principium, 26Q, 76va }\end{array}$ \\
\hline Physica & $\begin{array}{l}\text { P, 1ra ; VIII, 3Q, 8ra ; V, 7E, 17rb ; III, 16E, 39va ; III, 16E, 39va; } \\
\text { VII, 18E, 45rb ; III, 23E, 61va ; 25E, 69vb ; I, circa finem, 26E, } \\
\text { 73ra ; V, 26E, 74ra ; 29E, 81vb ; VIII, 29E, 82ra ; III, 30E, 84ra } \\
\text { VIII, 30E, 85ra ; VII, 30E, 86rb }\end{array}$ \\
\hline Metaphysica & $\begin{array}{l}\text { VI, P, 1va ; IV, 1Q, 4va ; IX, 1Q, 4vb ; XII, 3E, 7rb ; III, 1, 3Q, 8va } \\
\text { IX, 7Q, 17vb ; XI, 8Q, 20va ; II, 9E, 23va ; III, 10Q, 26ra ; IV, 16E, } \\
\text { 39rb ; II, 16E, 40ra ; V, 18E, 44rb ; I, 21E, 54va ; IX, 21E, 54vb } \\
\text { VIII, 21E, 54vb ; XII, 23E, 60ra ; in principio, 23E, 61ra ; VIII, 25E, } \\
\text { 69ra ; II, 25E, 69rb ; IV, 26E, 75va ; VII, 29E, 82va ; VIII, 30E, } \\
\text { 84va ; in fine, 30Q, 87rb }\end{array}$ \\
\hline Ethica & VI, 19E, 47va ; IV, 24E, 66va ; 24Q, 66va \\
\hline Politica & 21E, 54ra ; 21E, 54rb ; 23E, 61ra \\
\hline
\end{tabular}

\begin{tabular}{|l|l|}
\hline \multicolumn{2}{|c|}{ 2. Bible - 38 occurrences } \\
\hline Genesis & $6,11 \mathrm{Q}, 27 \mathrm{va} ; 1,12 \mathrm{Q}, 29 \mathrm{va} ;$ in principio, 24E, 65vb \\
\hline Exodus (Moyses) & $10 \mathrm{Q}, 26 \mathrm{rb} ; 17 \mathrm{Q}, 43 \mathrm{rb} ; 15,22 \mathrm{Q}, 59 \mathrm{ra}$ \\
\hline Deuteronomium & $6,1 \mathrm{Q}, 4 \mathrm{vb}$ \\
\hline Psalmi & $1 \mathrm{Q}, 4 \mathrm{va} ; 22 \mathrm{Q}, 59 \mathrm{va} ; 24 \mathrm{E}, 64 \mathrm{rb} ; 24 \mathrm{E}, 65 \mathrm{vb}$ \\
\hline Proverbia & $20,22 \mathrm{Q}, 59 \mathrm{ra}$ \\
\hline Ecclesiastes & $3,1 \mathrm{Q}, 3 \mathrm{vb} ; 5,10 \mathrm{Q}, 26 \mathrm{ra} ; 3,29 \mathrm{Q}, 82 \mathrm{vb}$ \\
\hline Isaias & $45,1 \mathrm{Q}, 4 \mathrm{vb}$ \\
\hline Daniel & $3 \mathrm{Q}, 8 \mathrm{vb}$ \\
\hline Malachias & $1,1 \mathrm{Q}, 4 \mathrm{va}$ \\
\hline
\end{tabular}




\begin{tabular}{|l|l|}
\hline Secundum Lucam & $5,21 \mathrm{bQ}, 56 \mathrm{rb}$ \\
\hline Secundum Ioannem & $27,6 \mathrm{Q}, 16 \mathrm{rb} ; 15,6 \mathrm{Q}, 16 \mathrm{rb} ; 1,30 \mathrm{Q}, 87 \mathrm{va}$ \\
\hline Actus Apostolorum & $5,11 \mathrm{Q}, 27 \mathrm{vb} ; 30 \mathrm{Q}, 87 \mathrm{va}$ \\
\hline $\begin{array}{l}\text { secundum Aposto- } \\
\text { lum }\end{array}$ & $6 \mathrm{E}, 15 \mathrm{ra} ; 21 \mathrm{E}, 55 \mathrm{rb} ; 21 \mathrm{bQ}, 56 \mathrm{va}$ \\
\hline Ad Romanos & $11,1 \mathrm{Q}, 3 \mathrm{vb} ; 1,6 \mathrm{Q}, 16 \mathrm{ra} ; 20 \mathrm{Q}, 53 \mathrm{vb} ; 1,21 \mathrm{aQ}, 55 \mathrm{vb}$ \\
\hline Ad Corinthios I & $7,1 \mathrm{Q}, 5 \mathrm{ra} ; 13,6 \mathrm{Q}, 16 \mathrm{ra}-\mathrm{b}(2 \mathrm{x}) ; 13,21 \mathrm{aQ}, 56 \mathrm{ra}$ \\
\hline Ad Hebreos & $30 \mathrm{E}, 85 \mathrm{va}$ \\
\hline Epistula Iacobi & $1,1 \mathrm{Q}, 4 \mathrm{va}$ \\
\hline Apocalipsis & $5 \mathrm{Q}, 14 \mathrm{rb}$ \\
\hline
\end{tabular}

\begin{tabular}{|c|c|}
\hline \multicolumn{2}{|r|}{ 3. Augustinus - 30 occurrences } \\
\hline Sans autre référence & $\begin{array}{l}2 \mathrm{Q}, 6 \mathrm{va} ; 7 \mathrm{Q}, 18 \mathrm{vb} ; 7 \mathrm{Q}, 21 \mathrm{va} ; 11 \mathrm{Q}, 28 \mathrm{rb} ; 17 \mathrm{Q}, 43 \mathrm{rb} ; 18 \mathrm{Q}, 46 \mathrm{rb} ; \\
21 \mathrm{aQ}, 56 \mathrm{ra} ; 25 \mathrm{Q}, 71 \mathrm{va} ; 29 \mathrm{Q}, 82 \mathrm{vb} ; 30 \mathrm{Q}, 87 \mathrm{va}\end{array}$ \\
\hline Super Genesim & II, 3Q, 8rb ; 12Q, 29rb \\
\hline $\begin{array}{l}\text { Ennarationes in Psal- } \\
\text { mos }\end{array}$ & $25 \mathrm{Q}, 70 \mathrm{vb}$ \\
\hline Enchiridion & $3 \mathrm{Q}, 8 \mathrm{rb} ; 23 \mathrm{Q}, 63 \mathrm{ra}$ \\
\hline De Trinitate & $\begin{array}{l}\text { 7Q, 19ra ; III, 11Q, 28ra ; 15E, 35vb (2x) ; III, 16E, 39va ; I, 21aQ, } \\
55 \mathrm{vb} \text {; VIII, 4, 22Q, 59va ; III, 30E, 85vb }\end{array}$ \\
\hline Confessiones & 7Q, 18vb ; XII, 7Q, 19ra ; XII, 9E, 22rb ; 19Q, 48vb \\
\hline De civitate Dei & $\mathrm{XIX}, 3 \mathrm{Q}, 8 \mathrm{rb} ; 7 \mathrm{Q}, 17 \mathrm{vb}$ \\
\hline Retractaciones & $21 \mathrm{aQ}, 56 \mathrm{rb}$ \\
\hline
\end{tabular}

\begin{tabular}{|l|l|}
\hline \multicolumn{2}{|c|}{ 4. Dyonisius - 26 occurrences } \\
\hline Sans autre référence & $1 \mathrm{Q}, 3 \mathrm{vb} ; 3 \mathrm{E}, 7 \mathrm{va} ; 3 \mathrm{Q}, 8 \mathrm{va} ; 8 \mathrm{Q}, 21 \mathrm{vb} ; 14 \mathrm{Q}, 34 \mathrm{ra} ; 19 \mathrm{E}, 47 \mathrm{vb} ; 19 \mathrm{Q}$, \\
& $50 \mathrm{ra} ; 21 \mathrm{aQ}, 56 \mathrm{ra}(2 \mathrm{x}) ; 22 \mathrm{Q}, 59 \mathrm{ra} ; 30 \mathrm{E}, 84 \mathrm{vb}$ \\
\hline De divinis nominibus & $13,1 \mathrm{Q}, 4 \mathrm{vb} ; 7,3 \mathrm{Q}, 8 \mathrm{va} ; 10,3 \mathrm{Q}, 9 \mathrm{rb} ; 2,6 \mathrm{E}, 15 \mathrm{va} ; 10,7 \mathrm{Q}, 17 \mathrm{vb} ; 7$, \\
& 8Q, 20va 4, 8Q, 20va $18 \mathrm{E}, 45 \mathrm{ra} ; 1,22 \mathrm{Q}, 59 \mathrm{ra} ; 22 \mathrm{Q}, 59 \mathrm{va} ; 2,23 \mathrm{E}$, \\
& $62 \mathrm{ra} ; 4,24 \mathrm{E}, 64 \mathrm{vb}$ \\
\hline $\begin{array}{l}\text { De celestia hierar- } \\
\text { chia }\end{array}$ & $2,6 \mathrm{E}, 14 \mathrm{va} ; 2,7 \mathrm{E}, 16 \mathrm{vb} ; 12,10 \mathrm{Q}, 25 \mathrm{va}$ \\
\hline
\end{tabular}

\begin{tabular}{|l|l|}
\hline \multicolumn{2}{|c|}{ 5. Proclus -21 occurrences } \\
\hline Sans autre référence & $\begin{array}{l}\text { 2E, 6rb } ; 12 \mathrm{E}, 28 \mathrm{va} ; 15 \mathrm{E}, 35 \mathrm{ra} ; 15 \mathrm{E}, 35 \mathrm{rb} ; 17 \mathrm{E}, 41 \mathrm{va} ; 21 \mathrm{E}, 55 \mathrm{ra} ; \\
25 \mathrm{E}, 68 \mathrm{vb} ; 27 \mathrm{E}, 78 \mathrm{ra} ; 29 \mathrm{E}, 82 \mathrm{rb}\end{array}$ \\
\hline $\begin{array}{l}\text { Elementacio theolo- } \\
\text { gica }\end{array}$ & $\begin{array}{l}1,4 \mathrm{E}, 9 \mathrm{va} ; 194,14 \mathrm{E}, 32 \mathrm{va} ; 44,15 \mathrm{E}, 36 \mathrm{va}(2 \mathrm{x}) ; 111,19 \mathrm{E}, 47 \mathrm{r} ; \\
\left.\text { (n }{ }^{\circ} \text { de la proposition) }\right)\end{array}$ \\
\hline
\end{tabular}


6. Plato-14 occurrences

\begin{tabular}{|l|l|}
\hline $\begin{array}{l}\text { Sans autre référen- } \\
\text { ce (ydee separate) }\end{array}$ & $\begin{array}{l}1 \mathrm{Q}, 4 \mathrm{ra} ; 9 \mathrm{E}, 22 \mathrm{va} ; 9 \mathrm{Q}, 24 \mathrm{ra} ; 10 \mathrm{E}, 24 \mathrm{vb} ; 12 \mathrm{E}, 28 \mathrm{vb}(2 \mathrm{x}) ; 18 \mathrm{E}, 44 \mathrm{rb} \\
(3 \mathrm{x}) ; 19 \mathrm{E}, 46 \mathrm{vb} ; 22 \mathrm{E}, 57 \mathrm{va} ; 24 \mathrm{E}, 64 \mathrm{va} ; 26 \mathrm{E}, 73 \mathrm{ra}\end{array}$ \\
\hline Timeus & $9 \mathrm{Q}, 23 \mathrm{vb}$ \\
\hline
\end{tabular}

\begin{tabular}{|l|l|}
\hline \multicolumn{2}{|c|}{ 7. Commentator - 13 occurrences } \\
\hline Sans autre référence & 4E, 10vb; 13E, 31va ; 19E, 47rb ; 25E, 68vb ; 25Q, 71rb \\
\hline Super Metaphysicam & $\begin{array}{l}\text { III, 4Q, 12rb ; XII, 12E, 28va ; II, 13E, 30va ; XII, 14Q, 33vb ; IV, } \\
\text { 24E, 65va ; IX, 25E, 68rb ; VII, 30E, 85va-b }\end{array}$ \\
\hline Super Physicam & I, circa finem, 26E, 73ra \\
\hline
\end{tabular}

\begin{tabular}{|l|l|}
\hline \multicolumn{2}{|c|}{ 8. Boethius -11 occurrences } \\
\hline Sans autre référence & $1 \mathrm{Q}, 4 \mathrm{va} ; 1 \mathrm{Q}, 5 \mathrm{ra} ; 16 \mathrm{E}, 40 \mathrm{ra} ; 25 \mathrm{E}, 69 \mathrm{ra} ; 29 \mathrm{E}, 80 \mathrm{va}$ \\
\hline De consolacione & $18 \mathrm{E}, 45 \mathrm{rb} ; 20 \mathrm{Q}, 53 \mathrm{vb} ; 29 \mathrm{Q}, 83 \mathrm{rb}$ \\
\hline De Trinitate & $7 \mathrm{Q}, 17 \mathrm{vb}$ \\
\hline De unitate et uno & $7 \mathrm{Q}, 17 \mathrm{vb} ; 7 \mathrm{Q}, 19 \mathrm{va}$ (liber ille De unitate et uno non est Boethii) \\
\hline
\end{tabular}

\begin{tabular}{|l|l|}
\hline \multicolumn{2}{|c|}{ 9. Damascenus -8 occurrences } \\
\hline Sans autre référence & $\begin{array}{l}\text { 2Q, 6vb;3Q, 8rb } 3 \mathrm{Q}, 9 \mathrm{rb} ; 7 \mathrm{Q}, 17 \mathrm{vb} ; 7 \mathrm{Q}, 19 \mathrm{ra} ; 11 \mathrm{Q}, 28 \mathrm{ra} ; 16 \mathrm{E}, \\
\text { 40ra }\end{array}$ \\
\hline [De fide orthodoxa $]$ & II, 22E, 58vb \\
\hline
\end{tabular}

\begin{tabular}{|l|l|}
\hline \multicolumn{2}{|c|}{ 10. Gregorius -8 occurrences } \\
\hline Sans autre référence & $9 \mathrm{Q}, 24 \mathrm{ra} ; 11 \mathrm{Q}, 28 \mathrm{ra} ; 19 \mathrm{Q}, 49 \mathrm{vb} ; 26 \mathrm{Q}, 75 \mathrm{vb} ; 30 \mathrm{Q}, 87 \mathrm{rb}$ \\
\hline Dyalogi & $\mathrm{II}, 11 \mathrm{Q}, 27 \mathrm{vb}(2 \mathrm{x})$ \\
\hline $\begin{array}{l}\text { Omelia super Eze- } \\
\text { chiel }\end{array}$ & $\mathrm{I}, 11 \mathrm{Q}, 27 \mathrm{vb}-28 \mathrm{ra}$ \\
\hline
\end{tabular}

\begin{tabular}{|l|l|}
\hline \multicolumn{2}{|c|}{ 11. Anselmus -4 occurrences } \\
\hline Sans autre référence & 20Q, 53rb; 24E, 63va ; 24E, 64rb \\
\hline Monologion & $64,22 \mathrm{Q}, 59 \mathrm{va}$ \\
\hline
\end{tabular}

\begin{tabular}{|l|l|}
\hline \multicolumn{2}{|c|}{ 12. Ysaac (Isaac Israeli) - 2 occurrences } \\
\hline De definitionibus & $19 \mathrm{E}, 48 \mathrm{ra} ; 25 \mathrm{E}, 69 \mathrm{vb}$ \\
\hline
\end{tabular}


13. Tholomeus -2 occurrences

\begin{tabular}{l|l} 
Sans autre référence & $4 \mathrm{E}, 11 \mathrm{rb} ; 20 \mathrm{Q}, 83 \mathrm{rb}$
\end{tabular}

\begin{tabular}{|l|l|}
\hline \multicolumn{2}{|c|}{14 . Avicebro-1 occurrence } \\
\hline Fons vite & $7 \mathrm{Q}, 17 \mathrm{vb}$ \\
\hline
\end{tabular}

\begin{tabular}{|l|l|}
\hline \multicolumn{2}{|c|}{ 15. Avicenna -1 occurrence } \\
\hline Sans autre référence & $21 \mathrm{bQ}, 56 \mathrm{rb}$ \\
\hline
\end{tabular}

\begin{tabular}{|l|l|}
\hline \multicolumn{2}{|c|}{ 16. Basilius -1 occurrence } \\
\hline Sans autre référence & $3 \mathrm{Q}, 8 \mathrm{rb}$ \\
\hline
\end{tabular}

17. Ysidorus -1 occurrence

\begin{tabular}{|l|l} 
Sans autre référence & $8 \mathrm{Q}, 21 \mathrm{va}$
\end{tabular}

18. Yeronimus -1 occurrence

\begin{tabular}{l|l} 
Sans autre référence & $3 \mathrm{Q}, 8 \mathrm{rb}$
\end{tabular}

Relevons de cet ensemble de données quelques points essentiels déjà évoqués: l'écrasante somme de citations aristotéliciennes; l'importance exceptionnelle des sources théologiques; l'élaboration d'une lecture néoplatonicienne chrétienne (dionysienne), certainement d'inspiration thomasienne; la présence non-négligeable des sources arabes, probablement d'origine albertinienne, souvent transmise par le biais du commentaire de Gilles de Rome.

\section{Le prologue}

Le prologue (Cod. Borgh. 352, f. 1rb-vb) ${ }^{105}$, qui suit le libellé de la première proposition du Liber de causis, présente une analyse générale du texte commenté, selon les critères habituellement retenus dans un accessus ad auctorem. Guillaume de Leus, en suivant de près une partie du Prohemium de Gilles de Rome ${ }^{106}$, discute ainsi du sujet de cette œuvre (materia sive su-

${ }^{105}$ Voir ci-dessous, en Annexe 2, l'édition de ce texte.

${ }^{106}$ Voir Aegidius Romanus, Super Librum de Causis, Venezia : J. Zoppin 1550, réimpr. Frankfurt a.M. 1968, Prohemium, M-Z. 
biectum), de son appartenance à un domaine spécifique de la philosophie (cui parti philosophie supponitur), de son auteur (causa efficiens), de sa forme (modus agendi sive forma tractandi), de son but (causa finalis) et de son titre (titulus).

Pour définir la materia du Liber de causis, le dominicain commence par affirmer, sous l'autorité d'Aristote, qu'opus nature est opus intelligencie $(f .1 \mathrm{va})^{107}$. En effet, selon lui, l'inférieur dépend, premièrement, du supérieur; or la nature est inférieure à l'intelligence. La nature agit, deuxièmement, en vue de sa fin, laquelle ne peut être conçue véritablement que par une intelligence. Ainsi, selon Guillaume, l'auteur du Liber, partant de ce présupposé, compare, dès la première proposition, l'influence de l'intelligence et celle de la nature, à savoir respectivement celles des premières causes séparées et celle de la cause seconde universelle, pour ensuite, dans les propositions suivantes, se concentrer sur les causes premières séparées, matière principale de ce traité.

Puisque l'auteur traite des causes premières séparées, cela permet de déterminer, à la suite de Gilles de Rome ${ }^{108}$, la partie de la philosophie à laquelle appartient cet ouvrage : non pas la physique, ni la mathématique, mais la métaphysique ou même la science divine, partie la plus noble de la métaphysique, traitant de Dieu et des substances séparées.

La causa efficiens de l'œuvre, à savoir son auteur, exige un développement important, vu les nombreuses interrogations qui entourent son identité. En s'inspirant du commentaire d'Albert le Grand, Guillaume de Leus rapporte que quelques-uns attribuent cette œuvre à un certain «David le Juif ${ }^{109}$, alors que d'autres penchent pour Alfarabi. En effet, - comme l'a

${ }^{107}$ Voir supra, 316-17, la réflexion sur cet axiome et sa paternité albertinienne.

${ }^{108}$ Aegidius Romanus, Super Librum de Causis, Prohemium, Y-Z : « Ulterius forte dubitaret aliquis cui parti philosophie supponatur hec scientia. Dicendum quod cum tres sint modi essenciales philosophie, ut dicitur sexto Metaphysice, videlicet physicus, mathematicus, et divinus seu methaphysicus, scientia tradita in hoc libro supponitur illi parti philosophie que dicitur divina sive methaphysica, et est quasi flos illius partis. Nam nobilissima pars methaphysice est considerare de Deo et de substantiis separatis ».

${ }^{109}$ Albertus Magnus, De causis et processu universitatis a Prima Causa II.1.1, 59. Albert le Grand ne fait pas de David le Juif un véritable auteur, mais plutôt un compilateur qui a arrangé sous forme de théorèmes des thèses d'Aristote, d'Avicenne, d'Algazel et d'Alfarabi. À propos de David le Juif et de sa possible identification avec le philosophe Avendauth et avec Abraham ibn Daud, voir M. TH. D'AlvernY, «Avendauth?», dans Homenaje Millàs Vallierosa, Barcelona 1954, 19-43, et M. ZonTA, « Jewish Mediation in the Translation of AraboIslamic Science and Philosophy to the Latin Middle Ages. Historical Overview and Perspectives of Research », dans Wissen über Grenzen. Arabisches Wissen und lateinisches Mittelalter, hrsg. v. A. SPEER und L. WEGENER (Miscellanea mediaeva- 
déjà démontré Thomas d'Aquin dans son commentaire, et comme le rapporte Gilles de Rome ${ }^{110}$, - ce livre n'a pas une origine grecque directe, mais a été traduit de l'arabe. En grec, l'on possède cependant les 211 propositions du platonicien Proclus intitulées Elementacio theologica, dont Guillaume s'ingénie à expliquer le titre en proposant une comparaison entre les éléments primordiaux (elementa), à la fondation de tous les autres composés (elementata) ${ }^{111}$ et de cette Elementacio theologica, au fondement de toutes les autres propositions de théologie. Concernant ces propositions grecques, Guillaume affirme, à la suite de Thomas d'Aquin, qu'elles sont à la source de celles qu'il s'apprête à commenter et qu'elles contiennent les mêmes vérités, mais de manière plus pleine et plus complète ${ }^{112}$. Il ajoute que, tout comme pour la Métaphysique et les livres naturels d'Aristote, il y eut une ancienne traduction en latin (dans ce cas, du Kalâm arabe par Gérard de Crémone au XII ${ }^{\mathrm{e}}$ siècle), puis une nouvelle réalisée dernièrement par un dominicain (de l'Elementacio theologica grecque, par Guillaume de Moerbeke en 1268). C'est aussi à partir de la lecture de cette nouvelle traduction (l'Elementacio theologica), explique Guillaume de Leus, qu'a été composé ce commentaire.

Le modus agendi (ou forma tractandi) de l'œuvre en question est défini, à la suite d'Albert et de Gilles de Rome ${ }^{113}$, comme une organisation par

lia 33), Berlin 2006, 89-105, 91, 101.

${ }^{110}$ Aegidius Romanus, Super Librum de Causis, Prohemium, M : «Causa vero efficiens ignoratur, sed creditur a multis fuisse Alpharabium. Dicitur enim quod hic liber non habetur apud Graecos, sed de Arabico dicitur esse translatus. Unde aliquis Arabs creditur fuisse author huius libri. In greco autem habentur propositiones Procli, a quibus hee propositiones emanaverunt et fuerunt accepte. Ut enim apparet scientibus : hic liber emanavit ab illo ».

${ }^{111}$ Pour les notions d'elementa et d'elementata selon Guillaume de Leus, voir BAV, Cod. Borgh. 352, ff. 43ra-44ra, par exemple f. 43rb : «elementa sunt eis [elementatis que sunt composiciora] simpliciora.... Duo sunt extrema simplicia in entibus quorum unum est actus purus, qui est Deus, aliud est pura potencia, que est materia »; f. 43vb : «patet quod elementa sunt magis vicina materie prime quam elementata $»$.

112 Cf. Thomas de Aquino, Super Librum de causis expositio, Prooemium, 3 : « Et in graeco quidem invenitur sic traditus liber Procli Platonici, continens ccxi propositiones, qui intitulatur Elementatio theologica; in arabico vero invenitur hic liber qui apud Latinos De causis dicitur, quem constat de arabico esse translatum et in graeco penitus non haberi : unde videtur ab aliquo philosophorum arabum [sic] ex praedicto libro Procli excerptus, praesertim quia omnia in hoc libro continentur, multo plenius et diffusuis continentur in illo ».

${ }^{113}$ Cf. Aegidius Romanus, Super Librum de Causis, Prohemium, N : « Modus autem agendi sive forma tractandi huius libri est per modum theorematum, quia primo proponuntur theoremata, et quasi quedam maxime universales, et postea datur intelleccio illorum theorematum, sive illarum proposicionum. Forma autem tractatus est ordinatio theorematum ad invicem $»$. 
théorèmes ou propositions (modus/ordinacio theorematum) : des théorèmes sont proposés par l'auteur comme des maximes universelles, puis sont exposées dans un commentaire.

Le but (causa finalis) consiste à permettre la connaissance de tout ce qui est transmis par le Liber de causis ${ }^{114}$.

Le titulus demande des explications plus fournies. En effet, ce dernier possède une quadruple forme, ainsi que l'avait présenté Gilles de Rome, selon une certaine interprétation du texte d'Albert le Grand ${ }^{115}$. On appelle communément cette œuvre Liber de causis, puisqu'on s'y occupe des causes premières dont dépendent les causes secondaires. Mais on le nomme aussi Flores divinorum, Lumen luminum et De pura bonitate. Flores divinorum, car il traite de la part la plus noble de la théologie, qui est la science divine; Lumen luminum, puisque, comme toute science peut être appelée lumière de l'âme, celle qui s'occupe des causes premières pourra être nommée lumière des lumières; De pura bonitate, parce que ce traité s'occupe de ce qui est abstrait, pur et non mélangé aux corps et qui possède une bonté indéfectible.

Si les philosophes ont placé dans la connaissance de ces objets l'ultime félicité, ceux-ci, rappelle le commentateur, ne nous sont connus non par leurs causes, mais seulement par leurs effets matériels. Il n'y a donc pas de

${ }^{114}$ Cf. Aegidius Romanus, Super Librum de Causis, Prohemium, N : « Causa autem finalis est cognicio eorum, que hic tractantur. Nam cognicio subiecti uno modo est precognicio in scientia, alio modo est finis scientie ».

${ }^{115}$ Guillaume reprend ici les informations fournies par AEGIDIUS RomANUS, Super Librum de Causis, Prohemium, O-P : «Titulus autem huius libri quadrupliciter ponitur. Nam uno modo intitulatur, quod est de pura bonitate. Alio modo intitulatur, quod hic liber vocatus est flores divinorum. Tertius titulus est, quod vocatus est lumen luminum. Quartus autem titulus eius est communis, quod dictus est liber de causis ; qui tituli forte ex dignitatibus, quas assignavimus huic libro sumpserunt originem. Dicebatur enim, quod hic liber maxime facit ad scire et quod per hunc librum habetur felicitas illa ultima, quam posuerunt phylosophi, si sufficienter esset hic liber traditus. Aspicientes ergo ad ipsum scire, vocaverunt ipsum de causis, eo quod scire sit per causas. Et vocaverunt ipsum lumen luminum, ut intelligamus, quod quelibet scientia est quasi quoddam lumen anime, sed scientia primarum causarum, et primorum principiorum (eo quod sit scientia scientiarum, et eo quod talis scientia faciat ad scire omnium scientiarum) huiusmodi scientia non solum debet vocari lumen, sed etiam lumen luminum. Sed aspicientes ad hanc doctrinam, prout habetur felicitas illa, quam posuerunt phylosophi, vocaverunt ipsum de pura bonitate, qui in bonitate pura, id est in bonitate abstracta, non autem in bonitate materiali, et corporali est ponenda felicitas. Sic etiam tales vocaverunt hunc librum Flores divinorum, qui in talibus vel in cognicione talium contemplativa felicitas (de qua hic loquimur) est ponenda ». Voir aussi Albertus Magnus, De causis et processu universitatis a Prima Causa II.1.1, 59. 
démonstration propter quid de ceux-ci, mais seulement quia, tout comme on ne possède pas la connaissance de quid sunt, mais seulement de quia sunt $t^{116}$.

Ainsi, conclut Guillaume de Leus, la réflexion qu'il s'apprête à engager à propos de ces causes premières, appartient à la plus haute science : «Ideo hec doctrina merito ascribit sibi nomen sapiencie » (f. 1vb).

Voici donc le résultat (provisoire) de nos premières études sur le dominicain Guillaume de Leus et son commentaire au Liber de causis. Lors d'une prochaine publication, nous reprendrons cette discussion qui nous permettra d'approfondir et d'élargir les recherches entreprises jusqu'à maintenant et de présenter son commentaire à la première proposition. Nous avons aussi le projet d'éditer l'intégralité du commentaire.

Comme support à l'article, nous proposons deux annexes. Tout d'abord, la liste des questions que pose Guillaume de Leus, associées à chacune des propositions du Liber de causis, puis l'édition, à partir du manuscrit BAV, Cod. Borgh. 352 (V) - qui est assez fautif -, de trois extraits de l'Exposicio in Librum de causis, à savoir l'épître dédicatoire au cardinal Pierre de la Chapelle-Taillefer, l'exposition de la méthode qui suit directement l'épître, ainsi que le prologue.

Nous avons choisi de reproduire l'orthographe médiévale, tout en l'harmonisant. Les mots entre parenthèses pointues sont ajoutés par l'éditeur. Le point d'interrogation entre crochets signifie une lectio incerta.

\section{Annexe 1 : Liste des questions associées aux propositions du Liber de causis ${ }^{117}$}

Prop. 1, ff. 1vb-3vb : Explicacio; ff. 3vb-5rb : Questio : queritur utrum sit dare tantum unam causam et unum principium omnium rerum.

Prop. 2, ff. 5rb-6rb : Explicacio; f. 6va-vb : Questio : queritur de hoc quod dicit actor hic, quod esse est plus quam eternitas et per consequens plus quam intelligencia.

\footnotetext{
${ }^{116}$ Cf. Aegidius Romanus, Super Librum de causis, Prohemium, S : « Dicendum quod de primis principiis et de primis causis non habemus scienciam, accipiendo scienciam sctricte, nec habemus scienciam simpliciter, et a priori, sed a posteriori, et per effectus tantum, de talibus enim habemus sapienciam et intellectum, que sunt cognicio pocior, et alcior quam sciencia et demonstracio ».

${ }^{117}$ La liste des questions a déjà été proposée par PELzER, « Guillaume de Leus (de Levibus) frère prêcheur de Toulouse », 403-4; nous l'avons corrigée en quelques endroits.
} 
Prop. 3, ff. 6vb-8rb : Explicacio; ff. 8rb-9va : Questio : queritur utrum corpora celestia sint animata.

Prop. 4, ff. 9va-11va : Explicacio; ff. 11va-12va : Questio : queritur de hoc quod dicit hic actor, scilicet utrum esse sit per creacionem.

Prop. 5, ff. 12va-13vb : Explicacio; ff. 13vb-14rb : Questio : queritur utrum motus celorum sit perpetuus uel cesset aliquando naturaliter.

Prop. 6, ff. 14rb-15va : Explicacio; ff. 15va-16va : Questio : queritur utrum prima causa possit hic sciri a nobis.

Prop. 7, ff. 16va-17vb : Explicacio; ff. 17vb-19va : Questio : queritur utrum angeli sint compositi ex ${ }^{118}$ materia et ex forma.

Prop. 8, ff. 19va-20va : Explicacio; ff. 20va-21vb : Questio : queritur utrum angeli cognoscant res per species rerum vel per essenciam suam.

Prop. 9, ff. 21vb-23vb : Explicacio; ff. 23vb-24ra : Questio : queritur $<\mathrm{u}-$ trum $>$ omnes intelligencie per naturam sint ita fixe et stabiles, quod sunt incorruptibiles.

Prop. 10, ff. 24ra-25va : Explicacio; ff. 25va-26vb : Questio : queritur utrum angeli superiores intelligant per species magis universales quam inferiores.

Prop. 11, ff. 26vb-27ra : Explicacio; ff. 27ra-28rb : Questio : queritur utrum intelligencia possit aliquo modo agere in ista corporalia vel corruptibilia inferiora.

Prop. 12, ff. 28ra-29rb : Explicacio; f. 29rb-vb : Questio : queritur utrum, sicut dicitur hic, omnia sint in omnibus.

Prop. 13, ff. 29vb-31vb : Explicacio; ff. 31vb-32ra : Questio : queritur utrum intelligencia, ex hoc quod intelligit se, intelligat omnia alia.

Prop. 14, ff. 32ra-33vb : Explicacio; ff. 33vb-34va : Questio : queritur utrum intelligencia superior possit immediate movere orbem.

Prop. 15, ff. 34va-36vb : Explicacio; ff. 36vb-37vb: Questio : queritur utrum sola res intellectualis convertat se supra se per operacionem ${ }^{119}$ intelligibilem.

Prop. 16, ff. 37vb-40va : Explicacio; ff. 40va-41rb : Questio : queritur utrum Deus immediate agat in omnibus rebus.

Prop. 17, ff. 41rb-43ra : Explicacio; ff. 43ra-44ra : Questio : queritur utrum res quanto sunt simpliciores tanto sint perfecciores.

Prop. 18, ff. 44ra-45vb : Explicacio; ff. 45vb-46va : Questio : queritur u-

\footnotetext{
118 ex] et $\mathrm{V}$

119 operacionem] et $a d d$. V
} 
trum attributa divina, cuiusmodi sunt esse, vivere et intelligere et quecumque alia attributa, differant in Deo realiter.

Prop. 19, ff. 46vb-48vb : Explicacio; ff. 48vb-50rb : Questio : queritur utrum intelligenciis sint gradus secundum sub et supra, sic quod superiores recipiant plus de bonitatibus divinis et per consequens sint perfecciores quam inferiores.

Prop. 20, ff. 50rb-53rb : Explicacio; f. 53rb-vb : Questio : queritur utrum prima causa se habeat uniformiter et equaliter ad omnia, ita quod tota causa diversitatis sit solum ex diversitate recipiencium.

Prop. 21, ff. 53vb-55va : Explicacio; ff. 55va-56rb : Questio prima : queritur utrum ex creaturis possimus devenire perscrutando ad cognicionem Dei ; $\mathrm{f}$. 56rb-56va : Questio secunda : queritur utrum quicquid ${ }^{120}$ Deus facit in creatura faciat ex nimia sui gracia vel ex debito vel ex merito creature.

Prop. 22, ff. 56va-59ra : Explicacio; f. 59ra-vb : Questio : queritur utrum prima causa possit hic a nobis aliquo modo proprie nominari.

Prop. 23, ff. 59vb-62va : Explicacio; ff. 62va-63rb : Questio : queritur utrum prima causa habeat universale regimen omnium rerum.

Prop. 24, ff. 63rb-66va : Explicacio; ff. 66va-68ra : Questio : queritur utrum Deus existat in omnibus rebus secundum disposicionem unam et omnia existant in Deo secundum variam disposicionem.

Prop. 25, ff. 68ra-70va : Explicacio; ff. 70va-71vb : Questio : queritur utrum in substanciis separatis sit generacio.

Prop. 26, ff. 71vb-75va : Explicacio; ff. 75va-76rb : Questio : queritur utrum substancie separate sint aliquo modo destructibiles.

Prop. 27, ff. 76rb-77rb : Explicacio; f. 77rb-vb : Questio : cum omnis forma in quantum forma adhereat materie sue et faciat unum cum materia secundum esse, ex quo largo modo omnis forma est delata super materiam suam, queritur ex hoc utrum omnis forma sit corruptibilis.

Prop. 28, ff. 78ra-79vb : Explicacio; ff. 79vb-80rb : Questio : queritur utrum, sicut omnis substancia per se stans est simplex et indivisibilis, ita etiam omnis substancia non stans per se sit divisibilis.

Prop. 29, ff. 80rb-82vb : Explicacio; ff. 82vb-83va : Questio : queritur utrum substancia simplex stans per se cadat sub tempore.

Prop. 30, ff. 83vb-87ra : Explicacio; f. 87ra-va : Questio : queritur utrum omnes partes universi sint connexe ad invicem inter se.

120 quicquid] in marg. V 


\section{Annexe 2 : Édition de l'épître dédicatoire, de l'exposition de la méthode et du prologue}

Reverendo et in cultu christiane religionis fideli devocione eximio, non V lra tam generis nobilitate quam morum honestate, preclaro domino Petro de

5 Capella largiente Deo, a quo bona cuncta procedunt, sacrosancte et romane Ecclesie venerabili cardinali devotus sibi in Christo frater Guillelmus de Liues, ordinis fratrum predicatorum, nacione Tolosanus, fructum aliqualem laboris sui studii sibi offerens, ex affectu vitam bonam et exitum beatum.

Quia, sicut notum est universis rectis ecclesiasticis vitam et statum ve10 strum intuentibus, vos estis sapiencie amator verus et sincerus, Dei dulcor necnon et studiosorum et studii fruens et diligens sublimator, idcirco attencius ego, considerans has non modicas graciarum distribuciones in vobis divinitus positas, destinavi in corde meo et operi mancipavi offerre sanctitati vestre de penuria studii mei exiguum munus, scilicet exposicionem 15 Libri de causis, in quo, quasi in libro omnino sapienciali, principaliter immo totaliter agitur de causis celestibus et divinis, super quo dominacioni vestre supplico magis pensare affectum meum quam censum, licet vos erga pauperes Christi non solum affectum, immo et censum quamplurimum laxando in sancta misericordie pietate solita incessanter et infatigabili cle20 mencia impendatis.

Circa exposicionem autem supradicti Libri de causis sic procedo : primo V 1ra premitto totam proposicionem de grossa littera textuali ; secundo immediate adiungo totum commentum illius proposicionis; postea vero expono per ordinem proposicionem et commentum cum propriis divisionibus et 25 multis adhuc eciam necessariis notabilibus; ultimo vero adnecto unam quescionem cum argumentis de difficiliori et utiliori puncto quod tangitur in illa proposicione vel commento eius, et sic deinceps prosequendo et continuando a prima proposicione libri huius usque ad ultimam proposicionem.

V = Città del Vaticano, BAV, Cod. Borgh. 352. L'épître dédicatoire et l'exposition de la méthode ont déjà été proposées par PELzER, «Guillaume de Leus (de Levibus) frère prêcheur de Toulouse », 400-1; nous les avons corrigées en quelques endroits.

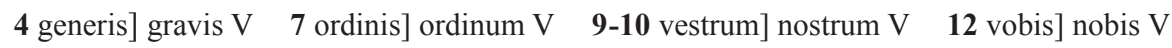
14 penuria] penu ac $\mathrm{V} \quad 15$ omnino] spatiali add. sed exp. V 17 vestre] nostre V | vos] igitur add. sed exp. V 21 de] et sed exp. V 23 commentum] convinctum V

5 Collecte du $5^{\mathrm{e}}$ dimanche après Pâques, qu'on retrouve dans Corpus orationum II, ed. E. Moeller, J.-M. Clément et B. Coppieters't Wallant (CCSL 150A), Turnhout 1993, oratio 1085 . 
V 1rb ...Sicut dicit PhiLosophus in libro Physicorum, due sunt cause entium, scilicet intellectus et natura, ita quod, secundum PHILOSOPHUM, opus na- 30 ture est opus intelligencie, id est quod natura habet totam efficaciam suam et virtutem productivam ex influencia intelligencie. Quod quidem oportet propter duo. Primo quia, cum inferius dependeat et recipiat a superiori, et intelligencia sit superior omni corporali natura, oportet opus nature reduci ad opus et virtutem intelligencie, quia quicquid potest inferior, potest virtus 35 superior. Secundo quia, secundum PHILOSOPHUM, natura agit propter finem, quod patet ex hoc, quia illa que conveniunt in natura specifica conveniunt eciam in fine naturali et in operacione per quam pertinguunt ad finem, sicut patet quod omnes aranee eodem modo faciunt telam et omnes yrundines eodem modo faciunt nidum ex eodem fine ab eis intento, scilicet 40 propter pullos ibi generandos et nutriendos. Quia igitur ista agencia corporalia intellectu carencia non possunt cognoscere ordinem nec proporcionem sui ad finem, indigent moveri et dirigi ad fines suos per agens intellectuale, $\mathrm{V}$ 1va sicut sagitta mittitur et dirigitur ad lignum a sagittante. Ex $\mid$ quo patet quod oportet totum opus nature fundari in influencia in<telligencie $>$.

Actor ergo Libri de causis hoc intelligens comparat hic in prima proposicione influenciam intelligencie ad virtutem nature, cum enim intendat hic agere de substanciis separatis et de primis causis. Que quidem cause non solum habent ordinem inter se, sed eciam respectu nature. Ideo, ut descendat ad principale intentum, scilicet ad causas primarias separatas, comparat 50 hic primo illas ad secundam causam universalem, que est natura, ut est ostensa differencia inter causas primarias separatas et causam secundam naturalem. Relicta causa secunda, convertat se ad tractandum de causis primariis separatis in sequentibus proposicionibus, ex quo patet materia huius operis sive subiectum.

Intendit enim actor hic tractare de causis primariis, ex quo patet cui parti philosophie supponatur, cum enim sint tres modi essentiales philosophie, ut dicitur $\mathrm{VI}^{\circ}$ Metaphysice, videlicet physici, qui tractat de hiis que sunt subiecta motui et materie ; et mathematici, qui tractat de hiis que sunt abstracta a motu, sed non a materia; et metaphysici, qui tractat de hiis que 60 sunt abstracta a motu et a materia. Patet quod doctrina ita supponitur illi

42 possunt] et add. $\mathrm{V} \mid$ cognoscere] cognosce V 44 lignum] sagitte add. sed exp. V | quo patet] quorum $\mathrm{V} \quad \mathbf{4 6}$ ergo] de add. sed exp. V 48 primis] et add. V

29 Aristoteles, Physica II c.4 (196a). 30 Voir à propos de cet axiome albertinien la discussion supra, 316-17. 36 Aristoteles, Physica II c.8 (199a). 58 Aristoteles, Metaphysica IX c.1 (1026a). 
parti philosophie, que dicitur divina sive metaphysica, et est quasi flos illius partis. Nam illa nobilissima pars metaphysice est consideracio de Deo et substanciis separatis, de quibus agit versus finem libro, licet in VII ${ }^{\circ}$ 65 determinet de substancia sensibili.

Causa vero efficiens huius operis ignoratur, licet dicatur fuisse DAVID IUDEUS a quibusdam, et ab aliis ALPHARABIUS Arabs. Hic enim liber non fuit inventus in greco, sed fuit translatus de arabico. Unde ALPHARABIUS Arabs dicitur fuisse actor huius libri a multis. In greco autem habentur 70 proposiciones PROCLI platonici CCXI, que intitulantur Elementacio theologica. Que quidem proposiciones dicuntur esse elementacio per quandam similitudinem ad elementa corporalia. Ita quod sicut in huiusmodi elementis corporalibus, quasi in quibusdam principiis et causis primitivis et simplicibus, quodammodo virtualiter continentur omnia elementata, quasi ab 75 ipsis elementis derivata, sic eciam tota theologia philosophorum virtualiter continetur in predictis proposicionibus, quasi in quibusdam theologie maximis, quod dico maxima virtute, licet maxima quantitate. Ad instar elementorum ex quibus constant elementata, sic eciam resolvuntur ad ipsa, quasi ad quedam illorum minima et simplicia, dicuntur ergo esse elementa80 cio theologica, quasi quedam elementalis accio et produccio, iuxta nomen suum, in quantum ex huiusmodi primis elementis theologie, cetera tradita per physicos theologicos[?] divinitatis ad illa, quasi ad quasdam maximas, resolvuntur, sicut conclusiones ad premissas. Sicut eciam in aliis scientiis, omnes conclusiones ad principia suarum scienciarum resolvuntur, sicut ab 85 ipsis derivantur, inferuntur et per ipsa probantur, sic et in proposito, de quibus iste proposiciones sunt accepte, que multo plenius et diffusius continentur in illo <libro $>$. Hee autem proposiciones cum commentis suis, | v 1vb sicut et Metaphysica ARISTOTELIS et libri naturales eiusdem, fuerunt translate antiqua translacione, postea vero translacione nova per unum fratrem 90 ordinis fratrum predicatorum. Supra qua nova translacione, ego feci istud scriptum et exposicionem.

Modus autem agendi et forma tractandi est per modum theorematum : ibi proponuntur distincta theoremata quasi quedam maxime universales, et postea exponuntur per commentum factum ab eodem qui fecit proposicio-

64 substanciis separatis] suppositis $\mathrm{V}$ 67 Alpharabius] Alpharabiisus sed corr. V $\mathbf{7 4}$ elementata] elementa $\mathrm{V} \quad \mathbf{7 6}$ continetur] continentur $\mathrm{V} \quad \mathbf{7 7}$ maxima $^{1}$ ] maximis $\mathrm{V}$ maxima $^{2}$ ] maximis $\mathrm{V} \quad \mathbf{7 8}$ sic] sicut $\mathrm{V} \quad \mathbf{8 4}$ sicut] in aliis scientiis add. sed exp. V $\mathbf{8 5}$ ipsa] ipsum V 87 in illo] illuc V

64 Cf. Città del Vaticano, BAV, Cod. Borgh. 352, ff. 17v-18r. 
nes. Forma autem tractatus est ordinacio theorematum.

Causa finalis est cognicio eorum que hic traduntur.

Titulus libri est quadruplex. Vocatur enim communiter Liber de causis, quia hic agitur de causis primariis, a quibus dependent cause secundarie et ideo anthonomastice dicitur Liber de causis. Dicitur eciam esse Flores divinorum, quia, sicut dictum est, tractat de hiis de quibus tractat nobilis- 100 sima pars metaphysice, que est sciencia divina. Intitulatur eciam Lumen luminum; cum enim quelibet sciencia sit lumen anime, sciencia primarum causarum potest dici lumen luminum, eo quod est sciencia scienciarum per excellenciam, inquantum facit ad declaracionem omnium scienciarum. Intitulatur eciam De pura bonitate, quia determinat de hiis que sunt abs- 105 tracta et pura et inmixta corporibus et habent bonitatem indeffectibilem.

Ita quod eciam in cognicione eorum que traduntur, hii PHILOSOPHI ponunt ultimam felicitatem; que quidem hic propter suam excellenciam non sunt nobis nota per causas, sed solum per effectus materiales ; propter quod de eis non sit demonstacio propter quid sunt, sed quia sunt, cum non sint 110 demonstrabilia per causas, sed solum per effectus equivocos et defficientes ab eis. Et ideo non possit facere sciri de causis suis de quibus agitur hic quid sunt, sed solum quia sunt, quia tamen agitur hic de altissimis et primariis causis. Ideo hec doctrina merito ascribit sibi nomen sapiencie.

99 dicitur] de add. sed exp. V

\author{
Delphine CARRON FAIVRE (Paris) \\ FNS-École des Hautes Études en Sciences Sociales \\ delphinecarron@gmail.com
}

\begin{abstract}
This article presents the unedited commentary on the Liber de causis by the Dominican Guillaume de Leus, who was active beteween the end of the thirteenth and the beginning of the fourteenth century and was promoted to Master of Theology in 1309. After a reconstruction of Guillaume's biography, the article provides a survey of his works and proposes their dates, with a special focus on his Exposicio in Librum de causis. The article contains a description of the manuscript (Città del Vaticano, BAV, Cod. Borgh. 352) that preserves the work. Finally, the article describes the commentary, its literary genre, scope, implicit and explicit sources and its prologue. An Appendix presents a list of questions that are associated with the individual propositions as well as an edition of the dedicatory letter, of the exposition of the method, and of the prologue.
\end{abstract}

Keywords: Dominicans, Guillaume de Leus, Liber de causis, Theology, Toulouse. 
